\title{
Power Improved Generalized Heronian Mean Operators Utilizing Hamacher Operations with Picture Fuzzy Information
}

\author{
Baolin Li (iD) and Lihua Yang (iD \\ School of Economics and Management, Hubei University of Automotive Technology, Shiyan 442002, Hubei, China \\ Correspondence should be addressed to Lihua Yang; 20159482@qq.com
}

Received 19 April 2021; Revised 6 June 2021; Accepted 24 June 2021; Published 16 July 2021

Academic Editor: Harish Garg

Copyright (C) 2021 Baolin Li and Lihua Yang. This is an open access article distributed under the Creative Commons Attribution License, which permits unrestricted use, distribution, and reproduction in any medium, provided the original work is properly cited.

\begin{abstract}
In multiple attribute decision-making (MADM), to better denote complicated preference information of decision-makers (DMs), picture fuzzy set (PFS) as an expansion of intuitionistic fuzzy set (IFS) has become a powerful tool in the recent years. Meanwhile, to remove the impact of abnormal data and capture the correlations among attributes in MADM issue, we propose the power improved generalized Heronian mean (PIGHM) operators in this paper, which have the merits of both power average (PA) operator and improved generalized Heronian mean (IGHM) operator. Additionally, Hamacher operations as a generalization of Algebraic operations and Einstein operations demonstrate good smooth approximate. Motivated by these, the main purpose is to explore PIGHM operators utilizing Hamacher operations to cope with MADM issue with picture fuzzy information. First, we introduce the Hamacher operations, the normalized hamming distance, and similarity measure of picture fuzzy numbers (PHNs). Second, based on these, two new picture fuzzy aggregating operators (AOs), the picture fuzzy Hamacher weighted power improved generalized Heronian mean (PFHWPIGHM) operator and the picture fuzzy Hamacher weighted geometric power improved generalized Heronian mean (PFHWGPIGHM) operator, are put forward, and some properties and special instances of proposed AOs are also investigated. Third, a new MADM model in terms of the PIGHM AOs is developed. Eventually, a practical MADM example, together with sensitivity analysis and comparative analysis, is conducted to verify the credibility and superiority of the new MADM model.
\end{abstract}

\section{Introduction}

Intuitionistic fuzzy set (IFS), which was firstly defined by Atanassov [1] and is an extended form of fuzzy set (FS) proposed by Zadeh [2], can better depict the truth, the falsity, and indeterminacy memberships, where the degree of indeterminacy membership is dependent on the membership degrees of truth and falsity. However, in actual environment, the indeterminacy degree should be given by decision-makers (DMs) according to real situation. For instance, in a voting, sixty percent are for it, ten percent remain neutral, twenty percent are against it, and ten percent refuse to vote. Clearly, this circumstance is beyond the capability of IFS. Thus, picture fuzzy set (PFS) defined by Cuong [3], including the degrees of positive, neutral, and negative, can convey multiple responses of DMs.
Since PFS can express different attitudes of DMs in complex situations, some studies regarding PFS have been conducted [4-9], concentrating on the aggregating operators (AOs), distance measure, cross entropy, and correlation coefficient. AOs, as an interesting direction owing to the diversity correlations among attributes, are an efficient tool for information fusion in MADM problem. Power average (PA) operator, which can decrease the impact of irrational data provided by DMs, was firstly defined by Yager [10]. Then, the PA operator has been applied to cope with different fuzzy information. Rani and Garg [11] applied PA operator and its extension to complex intuitionistic fuzzy set. Jiang et al. [12] proposed PA operator based on entropy and applied it to IFS. Ju et al. [13] extended PA AOs to q-rung orthopair fuzzy set (qROFS). Yang and Li [14] explored PA AOs to multivalued neutrosophic linguistic set 
(MVNLS). Garg and Kumar [15] constructed a MADM model utilizing PA AOs for linguistic IFS. Liu and Luo [16] extended PA AOs to simplified neutrosophic set (SNS). Garg and Nancy [17] developed PA AOs to linguistic singlevalued neutrosophic set (LSVNS). Liu et al. [7] applied PA AOs to complex PFS. Considering the interrelationships among aggregating values, Heronian mean (HM) operator was initially introduced by Beliakov et al. [18]. Later, Sykora [19] proposed the generalized HM (GHM) operator. HM operator and GHM operator mentioned above do not consider the weights of input argument. Then, $\mathrm{Yu}$ and $\mathrm{Wu}$ [20] extended the GHM to interval-valued IFS (IVIFS) and established generalized weighted HM (GWHM) operator. Moreover, $\mathrm{Yu}$ [21] introduced generalized geometric weighted HM (GGWHM) operator with IFS. Nevertheless, it seems to be counterintuitive that the GWHM and GGWHM operators do not have the characteristics of reducibility and idempotency. Therefore, Chu and Liu [22] proposed novel weighted HM AOs to uncertain linguistic information, namely, weighted generalized HM (WGHM) operator and weighted generalized geometric HM (WGGHM) operator, and proved the properties of reducibility, idempotency, monotonicity, and boundedness. Li et al. [23] further extended weighted HM AOs to SVNS and put forward improved generalized weighted HM (IGWHM) and improved generalized weighted geometric HM (IGWGHM) operators. Subsequently, IGWHM and IGWGHM operators are extended to neutrosophic hesitant fuzzy set (NHFS) [24], normal neutrosophic set (NNS) [25], neutrosophic uncertain linguistic set (NULS) [26], and bipolar neutrosophic set (BNS) [27].

Since each operator has its own merit, the combination of different operators has become a hotspot in MADM problem. Motivated by the superiorities of both PA operator and HM operator, Liu [28] combined them and proposed power Heronian (PH) operator and power weighted Heronian (PWH) operator to manage IVIFS. Later, Shi et al. [29] presented power geometric Heronian mean (PGHM) operator and weighted PGHM (WPGHM) operator to IFS. Zhao et al. [30] extended PH operator, weighted PH (WPH) operator, geometric PH (GPH) operator, and weighted GPH (WGPH) operator to SVNS. Jiang et al. [31] applied operators of PH, WPH, GPH, and WGPH to interval-valued dual HFS (IVDHFS). Ju et al. [32] combined PA and generalized HM operator and applied to fuse hesitant fuzzy linguistic numbers (HFLNs). Wang et al. [33] extended power generalized HM operator and its weighted form to q-rung orthopair hesitant fuzzy set (qROHFS). Moreover, rough PH operator [34] and power partitioned Heronian AOs [35, 36] have also been explored. As mentioned above, the combination of PA operator and HM operator has received the attention of many scholars. However, up to now, there is no research regarding the combination of PA operator and IGHM operator, which has more desirable properties than HM operator.

For aggregating PFS, different AOs have been explored in the recent years. Wang et al. [37] extended weighted Muirhead mean (WMM) and weighted dual MM (WDMM) to picture fuzzy set and proposed PFWMM and PFWDMM operator for financial investment risk appraisal. Jana et al. [38] established some Dombi AOs under picture fuzzy environment. Liu and Zhang [39] explored operator of A-PFLWAA to MADM under PFS. Zhang et al. [40] introduced Heronian mean (HM) operators using Dombi operations to PFS. Ates and Akay [41] expanded some Bonferroni mean (BM) to picture fuzzy set to handle MADM, including PFBM, PFNWBM, and PFOWBM operators. Luo and Xing [42] proposed picture fuzzy interaction partitioned Heronian (PFIPH) operator for selecting hotel. Qin et al. [43] introduced the Dempster-Shafer theory to PFS and proposed power Muirhead mean (PMM) operators utilizing the proposed operational rules. Yang and $\mathrm{Li}$ [9] defined multiple-valued picture fuzzy linguistic set (MVPFLS) and extended generalized HM operators to it. Up to date, AOs for PFS are almost based on Algebraic operations. Besides, there are a few researches on fusing picture fuzzy numbers (PFNs) utilizing Einstein [44], Dombi $[38,40]$, and Hamacher $[45,46]$. Hamacher operations, as an extension of Algebraic and Einstein operations, are more agile with a general parameter. Wang et al. [47] explored PA operator based on Hamacher to assess the quality of express service. However, in terms of the existing works, there is no research regarding exploring the combination of PA and IGHM operators to fuse picture fuzzy numbers (PFNs), especially using Hamacher operations.

In sum, the motivations of the paper are presented as follows:

(1) In complex situations, PFS is more suitable for expressing more answers of DMs, such as support, neutral, against, and refusal, which is beyond the capabilities of FS and IFS. Thus, PFS is selected to represent decision-making information in the paper.

(2) In more sophisticated cases, interrelationships among attributes are commonly existed in MADM issue, and unreasonable data may also be provided by DMs. Nevertheless, existing picture fuzzy AOs cannot reflect the correlations between attributes and alleviate the influence of abnormal data simultaneously. Thus, to solve this problem, the combination of both PA and IGHM operators is proposed in the paper.

(3) In MADM problem, to fuse information, aggregating operator is usually based on different operations, and Hamacher operations are more general and agile. Thus, it is an interesting topic to investigate the power improved generalized Heronian mean (PIGHM) AOs utilizing Hamacher operations.

Inspired by these factors, our contributions include the following: (1) We firstly combine PA and IGHM operators and propose some novel power improved generalized Heronian mean (PIGHM) AOs. (2) Based on Hamacher operations, we define novel weighted power improved generalized Heronian mean (WPIGHM) operator and weighted geometric power improved generalized Heronian mean (WGPIGHM) operator. (3) Based on the proposed AOs, we build a new MADM model to fuse PFNs. 
The framework of the paper is constructed as follows. In Section 2, the notion and Hamacher operations of PFNs as well as distance measure and cosine coefficient are introduced. In Section 3, PIGHM AOs utilizing Hamacher operations to picture fuzzy information are developed. Moreover, their predominant properties and particular instances are also analyzed. In Section 4, the MADM method based on PIGHM AOs with PFS is proposed. In Section 5, example and analysis are performed to verify the reliability of the model. In Section 6, some remarks are provided.

\section{Preliminaries}

Here, PFS and its Hamacher operations are introduced, and some measures and related operators are investigated as well.

\subsection{PFS}

Definition 1 (see [3]). A PFS $H$ in $Z$ is denoted as $H=\left\{\left\langle z, \mu_{H}(z), \eta_{H}(z), v_{H}(z)\right\rangle, \mid z \in Z\right\}$, where $\mu_{H}(z) \in$ $[0,1], \quad \eta_{H}(z) \in[0,1]$, and $\nu_{H}(z) \in[0,1]$ represent the positive, neutral, and negative memberships, respectively, meeting $0 \leq \mu_{H}(z)+\eta_{H}(z)+\nu_{H}(z) \leq 1, \quad \forall z \in Z, \quad$ and $\pi_{H}(z)=1-\mu_{H}(z)-\eta_{H}(z)-\nu_{H}(z)$ is called the refusal membership degree.

Particularly, if there is only one element in $Z, H$ is defined by $H=\left\langle\mu_{H}, \eta_{H}, v_{H}\right\rangle$, which is called picture fuzzy number (PFN).

2.2. Hamacher. Hamacher operations contain Hamacher product $\quad e \otimes f=(e f /(\gamma+(1-\gamma)(e+f-e f))) \quad$ and Hamacher sum $e \oplus f=(e+f-e f-(1-\gamma) e f /(1-(1-\gamma)$ $e f)$ ). Some operations of PFNs utilizing Hamacher are presented below.

Definition 2 (see [45]). Let $H=\left\langle\mu_{H}, \eta_{H}, \nu_{H}\right\rangle$ and $G=\left\langle\mu_{G}, \eta_{G}, \nu_{G}\right\rangle$ be two PFNs, and $\lambda>0$. The Hamacher operations of PFNs are defined as follows:

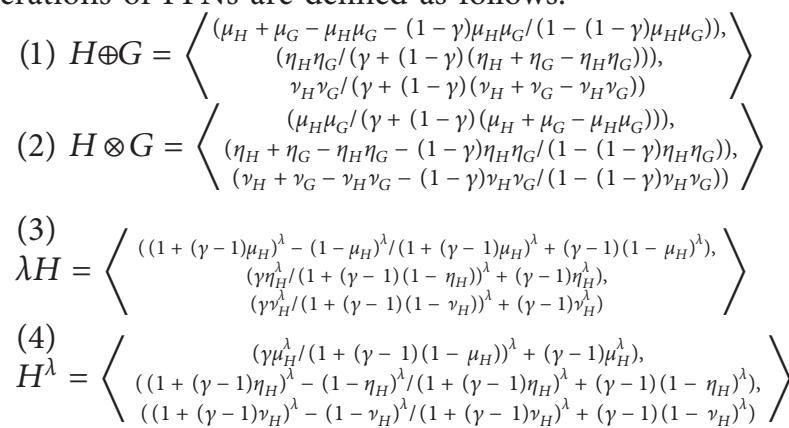

\subsection{Measure}

Definition 3. The normalized Hamming distance measure between two PFNs $H=\left\langle\mu_{H}, \eta_{H}, \nu_{H}\right\rangle$ and $G=\left\langle\mu_{G}, \eta_{G}, v_{G}\right\rangle$ is denoted by

$$
d(H, G)=\frac{1}{2}\left(\left|\mu_{H}-\mu_{G}\right|+\left|\eta_{H}-\eta_{G}\right|+\left|\nu_{H}-\nu_{G}\right|\right)
$$

where $d(H, G)$ meets these conditions: $0 \leq d(H, G) \leq 1$ and $d(H, G)=d(G, H)$.

The smaller the distance between two PFNs is, the bigger the similarity between them is.

Definition 4. The similarity measure between two PFNs $H=$ $\left\langle\mu_{H}, \eta_{H}, \nu_{H}\right\rangle$ and the ideal value $H^{*}=\langle 1,0,0\rangle$ is defined by

$$
s\left(H, H^{*}\right)=\frac{\mu_{H}}{\sqrt{\mu_{H}^{2}+\eta_{H}^{2}+v_{H}^{2}}} .
$$

\section{4. $P A$ and IGHM}

Definition 5. Let $\beta_{f}(f=1,2, \ldots, h)$ be multiple aggregated values, and the power average (PA) operator [10] and power geometric (PG) operator [48] are, respectively, denoted as

$$
\begin{aligned}
& \operatorname{PA}\left(\beta_{1}, \beta_{2}, \ldots, \beta_{h}\right)=\frac{\sum_{f=1}^{h}\left(1+\varphi\left(\beta_{f}\right)\right) \beta_{f}}{\sum_{f=1}^{h}\left(1+\varphi\left(\beta_{f}\right)\right)}, \\
& \operatorname{PG}\left(\beta_{1}, \beta_{2}, \ldots, \beta_{h}\right)=\prod_{f=1}^{h} \beta_{f}^{\left(1+\varphi\left(\beta_{f}\right) / \sum_{f=1}^{h}\left(1+\varphi\left(\beta_{f}\right)\right)\right),}
\end{aligned}
$$

where $\varphi\left(\beta_{f}\right)=\sum_{g=1, g \neq f}^{h} \sup \left(\beta_{f}, \beta_{g}\right)$ and $\sup \left(\beta_{f}, \beta_{g}\right)=1-$ $d\left(\beta_{f}, \beta_{g}\right)$ is the support between $\beta_{f}$ and $\beta_{g}$, which meets the following conditions:
(1) $\sup \left(\beta_{f}, \beta_{g}\right) \in[0,1]$
(2) $\sup \left(\beta_{f}, \beta_{g}\right)=\sup \left(\beta_{g}, \beta_{f}\right)$
(3) $\sup \left(\beta_{f}, \beta_{g}\right) \geq \sup \left(\beta_{f^{\prime}}, \beta_{g^{\prime}}\right)$

If $d\left(\beta_{f}, \beta_{g}\right) \leq d\left(\beta_{f^{\prime}}, \beta_{g^{\prime}}\right)$, here, $d\left(\beta_{f}, \beta_{g}\right)$ is the Hamming distance presented in Definition 3 .

Definition 6. Let $s, t \geq 0$ and $\beta_{f}(f=1,2, \ldots, h)$ be multiple aggregated values, and let $\omega=\left(\omega_{1}, \omega_{2}, \ldots, \omega_{h}\right)$ be a corresponding weight vector of $\beta_{f}(f=1,2, \ldots, h)$, where $\omega_{f} \geq 0$ and $\sum_{f=1}^{h} \omega_{f}=1$. Then, the improved generalized weighted Heronian mean (IGWHM) operator [23] and improved generalized geometric weighted Heronian mean (IGGWHM) operator [23] are, respectively, denoted as 


$$
\begin{aligned}
\operatorname{IGWHM}^{s, t}\left(\beta_{1}, \beta_{2}, \ldots, \beta_{h}\right) & =\left(\frac{\sum_{f=1}^{h} \sum_{g=f}^{h} \omega_{f} \omega_{g} \beta_{f}^{s} \beta_{g}^{t}}{\sum_{f=1}^{h} \sum_{g=f}^{h} \omega_{f} \omega_{g}}\right)^{(1 /(s+t))}, \\
\operatorname{IGGWHM}^{s, t}\left(\beta_{1}, \beta_{2}, \ldots, \beta_{h}\right) & =\frac{1}{s+t} \prod_{f=1}^{h} \prod_{g=f}^{h}\left(s \beta_{f}+t \beta_{g}\right)^{(2(h+1-f) / h(h+1)) \cdot\left(\omega_{g} / \sum_{k=f}^{h} \omega_{k}\right)},
\end{aligned}
$$

where the IGWHM operator has idempotency, monotonicity, and boundedness, and IGGWHM operator has reducibility, idempotency, monotonicity, and boundedness.

\section{Power Improved Generalized Heronian Mean Operators}

Based on Hamacher operations, two novel AOs, namely, the picture fuzzy Hamacher weighted power improved generalized Heronian mean (PFHWPIGHM) operator and the picture fuzzy Hamacher weighted geometric power improved generalized Heronian mean (PFHWGPIGHM) operator, are established. Besides, their properties and particular cases are explored as well.

\subsection{PFHWPIGHM Operator}

Definition 7. Let $s, t \geq 0$ and $\beta_{f}=\left\langle\mu_{f}, \eta_{f}, \nu_{f}\right\rangle$ $(f=1,2, \ldots, h)$ be multiple PFNs, and the importance of PFN $\beta_{f}$ is represented by $\omega_{f}$, meeting $\omega_{f} \geq 0$ and $\sum_{f=1}^{h} \omega_{f}=1$. Then, the picture fuzzy Hamacher weighted power improved generalized Heronian mean (PFHWPIGHM) operator is denoted as

$$
\operatorname{PFHWPIGHM}^{s, t}\left(\beta_{1}, \beta_{2}, \ldots, \beta_{h}\right)=\left(\frac{\oplus_{f=1}^{h} \oplus_{g=f}^{h} \omega_{f} \omega_{g}\left(h \varepsilon_{f} \omega_{f} / \sum_{e=1}^{h} \varepsilon_{e} \omega_{e} \beta_{f}\right)^{s} \otimes\left(h \varepsilon_{g} \omega_{g} / \sum_{e=1}^{h} \varepsilon_{e} \omega_{e} \beta_{g}\right)^{t}}{\sum_{f=1}^{h} \sum_{g=f}^{h} \omega_{f} \omega_{g}}\right)^{(1 /(s+t))},
$$

where $\quad \varepsilon_{f}=\left(1+\varphi\left(\beta_{f}\right) / \sum_{e=1}^{h}\left(1+\varphi\left(\beta_{e}\right)\right)\right), \quad \varphi\left(\beta_{f}\right)=$ $\sum_{g=1, g \neq f}^{h} \sup \left(\beta_{f}, \beta_{g}\right)$, and $\sup \left(\beta_{f}, \beta_{g}\right)=1-d\left(\beta_{f}, \beta_{g}\right)$.

Theorem 1. Let $s, t \geq 0$ and $\beta_{f}=\left\langle\mu_{f}, \eta_{f}, v_{f}\right\rangle(f=$ $1,2, \ldots, h)$ be multiple PFNs, and the importance of PFN $\beta_{f}$ is represented by $\omega_{f}$, meeting $\omega_{f} \geq 0$ and $\sum_{f=1}^{h} \omega_{f}=1$. Based on Hamacher operations in Definition 2, the aggregating value of PFHWPIGHM operator in Definition 7 is still a PFN and is shown as follows:

$$
\begin{aligned}
& \operatorname{PFHWPIGHM}^{\text {s.t. }}\left(\beta_{1}, \beta_{2}, \ldots, \beta_{h}\right)=
\end{aligned}
$$

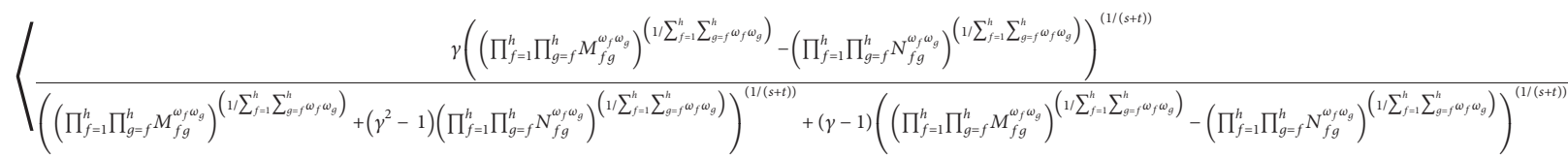

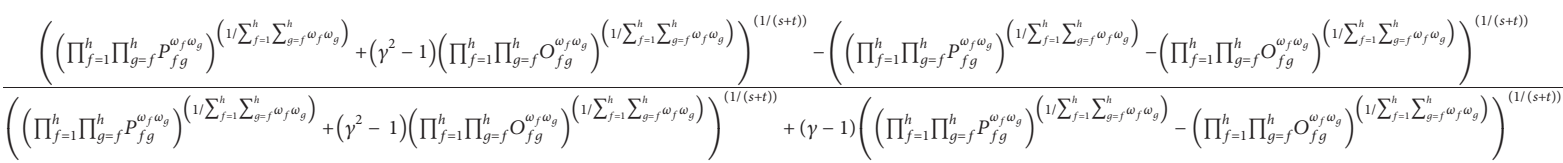

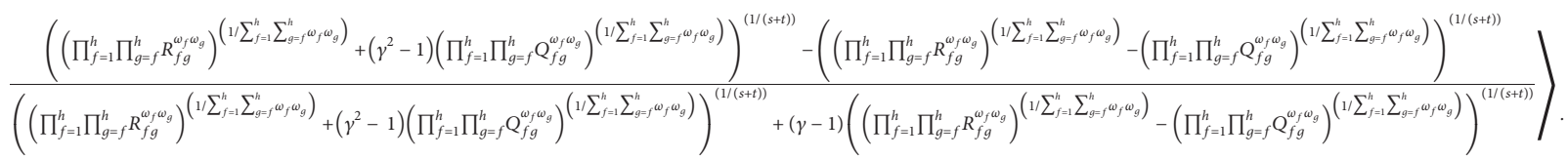

Here, 


$$
\begin{aligned}
& M_{f g}=\left(\left(1+(\gamma-1) \mu_{f}\right)^{h \zeta_{f}}+\left(\gamma^{2}-1\right)\left(1-\mu_{f}\right)^{h \zeta_{f}}\right)^{s}\left(\left(1+(\gamma-1) \mu_{g}\right)^{h \zeta_{g}}+\left(\gamma^{2}-1\right)\left(1-\mu_{g}\right)^{h \zeta_{g}}\right)^{t} \\
& +\left(\gamma^{2}-1\right)\left(\left(1+(\gamma-1) \mu_{f}\right)^{h \zeta_{f}}-\left(1-\mu_{f}\right)^{h \zeta_{f}}\right)^{s}\left(\left(1+(\gamma-1) \mu_{g}\right)^{h \zeta_{g}}-\left(1-\mu_{g}\right)^{h \zeta_{g}}\right)^{t} \\
& N_{f g}=\left(\left(1+(\gamma-1) \mu_{f}\right)^{h \zeta_{f}}+\left(\gamma^{2}-1\right)\left(1-\mu_{f}\right)^{h \zeta_{f}}\right)^{s}\left(\left(1+(\gamma-1) \mu_{g}\right)^{h \zeta_{g}}+\left(\gamma^{2}-1\right)\left(1-\mu_{g}\right)^{h \zeta_{g}}\right)^{t} \\
& -\left(\left(1+(\gamma-1) \mu_{f}\right)^{h \zeta_{f}}-\left(1-\mu_{f}\right)^{h \zeta_{f}}\right)^{s}\left(\left(1+(\gamma-1) \mu_{g}\right)^{h \zeta_{g}}-\left(1-\mu_{g}\right)^{h \zeta_{g}}\right)^{t}, \\
& O_{f g}=\left(\left(1+(\gamma-1)\left(1-\eta_{f}\right)\right)^{h \zeta_{f}}+\left(\gamma^{2}-1\right) \eta_{f}^{h \zeta_{f}}\right)^{s}\left(\left(1+(\gamma-1)\left(1-\eta_{g}\right)\right)^{h \zeta_{g}}+\left(\gamma^{2}-1\right) \eta_{g}^{h \zeta_{g}}\right)^{t} \\
& -\left(\left(1+(\gamma-1)\left(1-\eta_{f}\right)\right)^{h \zeta_{f}}-\eta_{f}^{h \zeta_{f}}\right)^{s}\left(\left(1+(\gamma-1)\left(1-\eta_{g}\right)\right)^{h \zeta_{g}}-\eta_{g}^{h \zeta_{g}}\right)^{t} \\
& P_{f g}=\left(\left(1+(\gamma-1)\left(1-\eta_{f}\right)\right)^{h \zeta_{f}}+\left(\gamma^{2}-1\right) \eta_{f}^{h \zeta_{f}}\right)^{s}\left(\left(1+(\gamma-1)\left(1-\eta_{g}\right)\right)^{h \zeta_{g}}+\left(\gamma^{2}-1\right) \eta_{g}^{h \zeta_{g}}\right)^{t} \\
& +\left(\gamma^{2}-1\right)\left(\left(1+(\gamma-1)\left(1-\eta_{f}\right)\right)^{h \zeta_{f}}-\eta_{f}^{h \zeta_{f}}\right)^{s}\left(\left(1+(\gamma-1)\left(1-\eta_{g}\right)\right)^{h \zeta_{g}}-\eta_{g}^{h \zeta_{g}}\right)^{t}, \\
& Q_{f g}=\left(\left(1+(\gamma-1)\left(1-v_{f}\right)\right)^{h \zeta_{f}}+\left(\gamma^{2}-1\right) v_{f}^{h \zeta_{f}}\right)^{s}\left(\left(1+(\gamma-1)\left(1-v_{g}\right)\right)^{h \zeta_{g}}+\left(\gamma^{2}-1\right) v_{g}^{h \zeta_{g}}\right)^{t} \\
& -\left(\left(1+(\gamma-1)\left(1-v_{f}\right)\right)^{h \zeta_{f}}-\nu_{f}^{h \zeta_{f}}\right)^{s}\left(\left(1+(\gamma-1)\left(1-v_{g}\right)\right)^{h \zeta_{g}}-v_{g}^{h \zeta_{g}}\right)^{t} \\
& R_{f g}=\left(\left(1+(\gamma-1)\left(1-\nu_{f}\right)\right)^{h \zeta_{f}}+\left(\gamma^{2}-1\right) \nu_{f}^{h \zeta_{f}}\right)^{s}\left(\left(1+(\gamma-1)\left(1-\nu_{g}\right)\right)^{h \zeta_{g}}+\left(\gamma^{2}-1\right) \nu_{g}^{h \zeta_{g}}\right)^{t} \\
& +\left(\gamma^{2}-1\right)\left(\left(1+(\gamma-1)\left(1-\nu_{f}\right)\right)^{h \zeta_{f}}-\nu_{f}^{h \zeta_{f}}\right)^{s}\left(\left(1+(\gamma-1)\left(1-\nu_{g}\right)\right)^{h \zeta_{g}}-\nu_{g}^{h \zeta_{g}}\right)^{t} \\
& \zeta_{f}=\frac{\varepsilon_{f} \omega_{f}}{\sum_{e=1}^{h} \varepsilon_{e} \omega_{e}}, \\
& \zeta_{g}=\frac{\varepsilon_{g} \omega_{g}}{\sum_{e=1}^{h} \varepsilon_{e} \omega_{e}} .
\end{aligned}
$$

Proof. Based on Hamacher operations of PFNs presented in Definition 2, we get 


$$
\begin{aligned}
& h \zeta_{f} \beta_{f}=\left\langle\frac{\left(1+(\gamma-1) \mu_{f}\right)^{h \zeta_{f}}-\left(1-\mu_{f}\right)^{h \zeta_{f}}}{\left(1+(\gamma-1) \mu_{f}\right)^{h \zeta_{f}}+(\gamma-1)\left(1-\mu_{f}\right)^{h \zeta_{f}}}, \frac{\gamma \eta_{f}^{h \zeta_{f}}}{\left(1+(\gamma-1)\left(1-\eta_{f}\right)\right)^{h \zeta_{f}}+(\gamma-1) \eta_{f}^{h \zeta_{f}}},\right. \\
& \left.\cdot \frac{\gamma \nu_{f}^{h \zeta_{f}}}{\left(1+(\gamma-1)\left(1-\nu_{f}\right)\right)^{h \zeta_{f}}+(\gamma-1) \nu_{f}^{h \zeta_{f}}}\right\rangle \\
& h \zeta_{g} \beta_{g}=\left\langle\frac{\left(1+(\gamma-1) \mu_{g}\right)^{h \zeta_{g}}-\left(1-\mu_{g}\right)^{h \zeta_{g}}}{\left(1+(\gamma-1) \mu_{g}\right)^{h \zeta_{g}}+(\gamma-1)\left(1-\mu_{g}\right)^{h \zeta_{g}}}, \frac{\gamma \eta_{g}^{h \zeta_{g}}}{\left(1+(\gamma-1)\left(1-\eta_{g}\right)\right)^{h \zeta_{g}}+(\gamma-1) \eta_{g}^{h \zeta_{g}}}\right. \\
& \left.\cdot \frac{\gamma \nu_{g}^{h \zeta_{g}}}{\left(1+(\gamma-1)\left(1-\nu_{g}\right)\right)^{h \zeta_{g}}+(\gamma-1) \nu_{g}^{h \zeta_{g}}}\right\rangle \\
& \left(h \zeta_{f} \beta_{f}\right)^{s}=\left\langle\frac{\gamma\left(\left(1+(\gamma-1) \mu_{f}\right)^{h \zeta_{f}}-\left(1-\mu_{f}\right)^{h \zeta_{f}}\right)^{s}}{\left(\left(1+(\gamma-1) \mu_{f}\right)^{h \zeta_{f}}+\left(\gamma^{2}-1\right)\left(1-\mu_{f}\right)^{h \zeta_{f}}\right)^{s}+(\gamma-1)\left(\left(1+(\gamma-1) \mu_{f}\right)^{h \zeta_{f}}-\left(1-\mu_{f}\right)^{h \zeta_{f}}\right)^{s}}\right. \\
& \left(\left(1+(\gamma-1)\left(1-\eta_{f}\right)\right)^{h \zeta_{f}}+\left(\gamma^{2}-1\right) \eta_{f}^{h \zeta_{f}}\right)^{s}-\left(\left(1+(\gamma-1)\left(1-\eta_{f}\right)\right)^{h \zeta_{f}}-\eta_{f}^{h \zeta_{f}}\right)^{s} \\
& \cdot \overline{\left(\left(1+(\gamma-1)\left(1-\eta_{f}\right)\right)^{h \zeta_{f}}+\left(\gamma^{2}-1\right) \eta_{f}^{h \zeta_{f}}\right)^{s}+(\gamma-1)\left(\left(1+(\gamma-1)\left(1-\eta_{f}\right)\right)^{h \zeta_{f}}-\eta_{f}^{h \zeta_{f}}\right)^{s}} \\
& \left.\cdot \frac{\left(\left(1+(\gamma-1)\left(1-\nu_{f}\right)\right)^{h \zeta_{f}}+\left(\gamma^{2}-1\right) \nu_{f}^{h \zeta_{f}}\right)^{s}-\left(\left(1+(\gamma-1)\left(1-v_{f}\right)\right)^{h \zeta_{f}}-\nu_{f}^{h \zeta_{f}}\right)^{s}}{\left(\left(1+(\gamma-1)\left(1-\nu_{f}\right)\right)^{h \zeta_{f}}+\left(\gamma^{2}-1\right) \nu_{f}^{h \zeta_{f}}\right)^{s}+(\gamma-1)\left(\left(1+(\gamma-1)\left(1-\nu_{f}\right)\right)^{h \zeta_{f}}-\nu_{f}^{h \zeta_{f}}\right)^{s}}\right\rangle, \\
& \left(h \zeta_{g} \beta_{g}\right)^{t}=\left\langle\frac{\gamma\left(\left(1+(\gamma-1) \mu_{g}\right)^{h \zeta_{g}}-\left(1-\mu_{g}\right)^{h \zeta_{g}}\right)^{t}}{\left(\left(1+(\gamma-1) \mu_{g}\right)^{h \zeta_{g}}+\left(\gamma^{2}-1\right)\left(1-\mu_{g}\right)^{h \zeta_{g}}\right)^{t}+(\gamma-1)\left(\left(1+(\gamma-1) \mu_{g}\right)^{h \zeta_{g}}-\left(1-\mu_{g}\right)^{h \zeta_{g}}\right)^{t}}\right. \\
& \cdot \frac{\left(\left(1+(\gamma-1)\left(1-\eta_{g}\right)\right)^{h \zeta_{g}}+\left(\gamma^{2}-1\right) \eta_{g}^{h \zeta_{g}}\right)^{t}-\left(\left(1+(\gamma-1)\left(1-\eta_{g}\right)\right)^{h \zeta_{g}}-\eta_{g}^{h \zeta_{g}}\right)^{t}}{\left(\left(1+(\gamma-1)\left(1-\eta_{g}\right)\right)^{h \zeta_{g}}+\left(\gamma^{2}-1\right) \eta_{g}^{h \zeta_{g}}\right)^{t}+(\gamma-1)\left(\left(1+(\gamma-1)\left(1-\eta_{g}\right)\right)^{h \zeta_{g}}-\eta_{g}^{h \zeta_{g}}\right)^{t}} \\
& \left.\frac{\left(\left(1+(\gamma-1)\left(1-\nu_{g}\right)\right)^{h \zeta_{g}}+\left(\gamma^{2}-1\right) \nu_{g}^{h \zeta_{g}}\right)^{t}-\left(\left(1+(\gamma-1)\left(1-v_{g}\right)\right)^{h \zeta_{g}}-\nu_{g}^{h \zeta_{g}}\right)^{t}}{\left.\left(1+(\gamma-1)\left(1-\nu_{g}\right)\right)^{h \zeta_{g}}+\left(\gamma^{2}-1\right) \nu_{g}^{h \zeta_{g}}\right)^{t}+(\gamma-1)\left(\left(1+(\gamma-1)\left(1-v_{g}\right)\right)^{h \zeta_{g}}-v_{g}^{h \zeta_{g}}\right)^{t}}\right\rangle .
\end{aligned}
$$

Let 


$$
\begin{aligned}
& A_{f}=\left(1+(\gamma-1) \mu_{f}\right)^{h \zeta_{f}}-\left(1-\mu_{f}\right)^{h \zeta_{f}}, \\
& B_{f}=\left(1+(\gamma-1) \mu_{f}\right)^{h \zeta_{f}}+\left(\gamma^{2}-1\right)\left(1-\mu_{f}\right)^{h \zeta_{f}}, \\
& C_{f}=\left(1+(\gamma-1)\left(1-\eta_{f}\right)\right)^{h \zeta_{f}}+\left(\gamma^{2}-1\right) \eta_{f}^{h \zeta_{f}}, \\
& D_{f}=\left(1+(\gamma-1)\left(1-\eta_{f}\right)\right)^{h \zeta_{f}}-\eta_{f}^{h \zeta_{f}}, \\
& E_{f}=\left(1+(\gamma-1)\left(1-v_{f}\right)\right)^{h \zeta_{f}}+\left(\gamma^{2}-1\right) \nu_{f}^{h \zeta_{f}}, \\
& F_{f}=\left(1+(\gamma-1)\left(1-v_{f}\right)\right)^{h \zeta_{f}}-v_{f}^{h \zeta_{f}}, \\
& A_{g}=\left(1+(\gamma-1) \mu_{g}\right)^{h \zeta_{g}}-\left(1-\mu_{g}\right)^{h \zeta_{g}}, \\
& B_{g}=\left(1+(\gamma-1) \mu_{g}\right)^{h \zeta_{g}}+\left(\gamma^{2}-1\right)\left(1-\mu_{g}\right)^{h \zeta_{g}}, \\
& C_{g}=\left(1+(\gamma-1)\left(1-\eta_{g}\right)\right)^{h \zeta_{g}}+\left(\gamma^{2}-1\right) \eta_{g}^{h \zeta_{g}}, \\
& D_{g}=\left(1+(\gamma-1)\left(1-\eta_{g}\right)\right)^{h \zeta_{g}}-\eta_{g}^{h \zeta_{g}}, \\
& E_{g}=\left(1+(\gamma-1)\left(1-v_{g}\right)\right)^{h \zeta_{g}}+\left(\gamma^{2}-1\right) \nu_{g}^{h \zeta_{g}}, \\
& F_{g}=\left(1+(\gamma-1)\left(1-v_{g}\right)\right)^{h \zeta_{g}}-v_{g}^{h \zeta_{g}} .
\end{aligned}
$$

Then,

$$
\begin{aligned}
& \left(h \zeta_{f} \beta_{f}\right)^{s} \otimes\left(h \zeta_{g} \beta_{g}\right)^{t}=\left\langle\frac{\gamma A_{f}^{s} A_{g}^{t}}{B_{f}^{s} B_{g}^{t}+(\gamma-1) A_{f}^{s} A_{g}^{t}}, \frac{C_{f}^{s} C_{g}^{t}-D_{f}^{s} D_{g}^{t}}{C_{f}^{s} C_{g}^{t}+(\gamma-1) D_{f}^{s} D_{g}^{t}}, \frac{E_{f}^{s} E_{g}^{t}-F_{f}^{s} F_{g}^{t}}{E_{f}^{s} E_{g}^{t}+(\gamma-1) F_{f}^{s} F_{g}^{t}}\right\rangle, \\
& \omega_{f} \omega_{g}\left(h \zeta_{f} \beta_{f}\right)^{s} \otimes\left(h \zeta_{g} \beta_{g}\right)^{t}=\left\langle\frac{\left(B_{f}^{s} B_{g}^{t}+\left(\gamma^{2}-1\right) A_{f}^{s} A_{g}^{t}\right)^{\omega_{f} \omega_{g}}-\left(B_{f}^{s} B_{g}^{t}-A_{f}^{s} A_{g}^{t}\right)^{\omega_{f} \omega_{g}}}{\left(B_{f}^{s} B_{g}^{t}+\left(\gamma^{2}-1\right) A_{f}^{s} A_{g}^{t}\right)^{\omega_{f} \omega_{g}}+(\gamma-1)\left(B_{f}^{s} B_{g}^{t}-A_{f}^{s} A_{g}^{t}\right)^{\omega_{f} \omega_{g}}},\right. \\
& \cdot \frac{\gamma\left(C_{f}^{s} C_{g}^{t}-D_{f}^{s} D_{g}^{t}\right)^{\omega_{f} \omega_{g}}}{\left(C_{f}^{s} C_{g}^{t}+\left(\gamma^{2}-1\right) D_{f}^{s} D_{g}^{t}\right)^{\omega_{f} \omega_{g}}+(\gamma-1)\left(C_{f}^{s} C_{g}^{t}-D_{f}^{s} D_{g}^{t}\right)^{\omega_{f} \omega_{g}}} \\
& \left.\cdot \frac{\gamma\left(E_{f}^{s} E_{g}^{t}-F_{f}^{s} F_{g}^{t}\right)^{\omega_{f} \omega_{g}}}{\left(E_{f}^{s} E_{g}^{t}+\left(\gamma^{2}-1\right) F_{f}^{s} F_{g}^{t}\right)^{\omega_{f} \omega_{g}}+(\gamma-1)\left(E_{f}^{s} E_{g}^{t}-F_{f}^{s} F_{g}^{t}\right)^{\omega_{f} \omega_{g}}}\right\rangle \\
& \underset{f=1}{\stackrel{\leftrightarrow}{\oplus} \underset{g=f}{h}} \omega_{f} \omega_{g}\left(h \zeta_{f} \beta_{f}\right)^{s} \otimes\left(h \zeta_{g} \beta_{g}\right)^{t}=\left\langle\frac{\prod_{f=1}^{h} \prod_{g=f}^{h}\left(B_{f}^{s} B_{g}^{t}+\left(\gamma^{2}-1\right) A_{f}^{s} A_{g}^{t}\right)^{\omega_{f} \omega_{g}}-\prod_{f=1}^{h} \prod_{g=f}^{h}\left(B_{f}^{s} B_{g}^{t}-A_{f}^{s} A_{g}^{t}\right)^{\omega_{f} \omega_{g}}}{\prod_{f=1}^{h} \prod_{g=f}^{h}\left(B_{f}^{s} B_{g}^{t}+\left(\gamma^{2}-1\right) A_{f}^{s} A_{g}^{t}\right)^{\omega_{f} \omega_{g}}+(\gamma-1) \prod_{f=1}^{h} \prod_{g=f}^{h}\left(B_{f}^{s} B_{g}^{t}-A_{f}^{s} A_{g}^{t}\right)^{\omega_{f} \omega_{g}}},\right. \\
& \gamma \prod_{f=1}^{h} \prod_{g=f}^{h}\left(C_{f}^{s} C_{g}^{t}-D_{f}^{s} D_{g}^{t}\right)^{\omega_{f} \omega_{g}}
\end{aligned}
$$

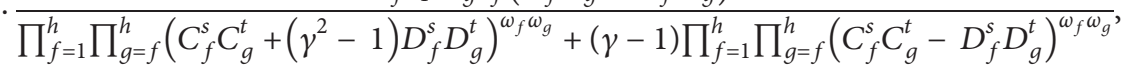

$$
\begin{aligned}
& \left.\cdot \frac{\gamma \prod_{f=1}^{h} \prod_{g=f}^{h}\left(E_{f}^{s} E_{g}^{t}-F_{f}^{s} F_{g}^{t}\right)^{\omega_{f} \omega_{g}}}{\prod_{f=1}^{h} \prod_{g=f}^{h}\left(E_{f}^{s} E_{g}^{t}+\left(\gamma^{2}-1\right) F_{f}^{s} F_{g}^{t}\right)^{\omega_{f} \omega_{g}}+(\gamma-1) \prod_{f=1}^{h} \prod_{g=f}^{h}\left(E_{f}^{s} E_{g}^{t}+\left(\gamma^{2}-1\right) F_{f}^{s} F_{g}^{t}\right)^{\omega_{f} \omega_{g}}}\right\rangle .
\end{aligned}
$$


Furthermore,

$$
\begin{aligned}
& \frac{\Theta_{f=1}^{h} \Theta_{g=f}^{h} \omega_{f} \omega_{g}\left(h \zeta_{f} \beta_{f}\right)^{s} \otimes\left(h \zeta_{g} \beta_{g}\right)^{t}}{\sum_{f=1}^{h} \sum_{g=f}^{h} \omega_{f} \omega_{g}} \\
& =\left\langle\frac{\left(\prod_{f=1}^{h} \prod_{g=f}^{h}\left(B_{f}^{s} B_{g}^{t}+\left(\gamma^{2}-1\right) A_{f}^{s} A_{g}^{t}\right)^{\omega_{f} \omega_{g}}\right)^{\left(1 / \sum_{f=1}^{h} \sum_{g=f}^{h} \omega_{f} \omega_{g}\right)}-\left(\prod_{f=1}^{h} \prod_{g=f}^{h}\left(B_{f}^{s} B_{g}^{t}-A_{f}^{s} A_{g}^{t}\right)^{\omega_{f} \omega_{g}}\right)^{1 / \sum_{f=1}^{h} \sum_{g=f}^{h} \omega_{f} \omega_{g}}}{\left(\prod_{f=1}^{h} \prod_{g=f}^{h}\left(B_{f}^{s} B_{g}^{t}+\left(\gamma^{2}-1\right) A_{f}^{s} A_{g}^{t}\right)^{\omega_{f} \omega_{g}}\right)^{1 / \sum_{f=1}^{h} \sum_{g=f}^{h} \omega_{f} \omega_{g}}+(\gamma-1)\left(\prod_{f=1}^{h} \prod_{g=f}^{h}\left(B_{f}^{s} B_{g}^{t}-A_{f}^{s} A_{g}^{t}\right)^{\omega_{f} \omega_{g}}\right)^{1 / \sum_{f=1}^{h} \sum_{g=f}^{h} \omega_{f} \omega_{g}}}\right. \\
& \quad \frac{\gamma\left(\prod_{f=1}^{h} \prod_{g=f}^{h}\left(C_{f}^{s} C_{g}^{t}-D_{f}^{s} D_{g}^{t}\right)^{\omega_{f} \omega_{g}}\right)^{1 / \sum_{f=1}^{h} \sum_{g=f}^{h} \omega_{f} \omega_{g}}}{\left(\prod_{f=1}^{h} \prod_{g=f}^{h}\left(C_{f}^{s} C_{g}^{t}+\left(\gamma^{2}-1\right) D_{f}^{s} D_{g}^{t}\right)^{\omega_{f} \omega_{g}}\right)^{1 / \sum_{f=1}^{h} \sum_{g=f}^{h} \omega_{f} \omega_{g}}+(\gamma-1)\left(\prod_{f=1}^{h} \prod_{g=f}^{h}\left(C_{f}^{s} C_{g}^{t}-D_{f}^{s} D_{g}^{t}\right)^{\omega_{f} \omega_{g}}\right)^{1 / \sum_{f=1}^{h} \sum_{g=f}^{h} \omega_{f} \omega_{g}}}, \\
& \left.\quad \cdot \frac{\gamma\left(\prod_{f=1}^{h} \prod_{g=f}^{h}\left(E_{f}^{s} E_{g}^{t}-F_{f}^{s} F_{g}^{t}\right)^{\omega_{f} \omega_{g}}\right)^{1 / \sum_{f=1}^{h} \sum_{g=f}^{h} \omega_{f} \omega_{g}}}{\left(\prod_{f=1}^{h} \prod_{g=f}^{h}\left(E_{f}^{s} E_{g}^{t}+\left(\gamma^{2}-1\right) F_{f}^{s} F_{g}^{t}\right)^{\omega_{f} \omega_{g}}\right)^{1 / \sum_{f=1}^{h} \sum_{g=f}^{h} \omega_{f} \omega_{g}}+(\gamma-1)\left(\prod_{f=1}^{h} \prod_{g=f}^{h}\left(E_{f}^{s} E_{g}^{t}+\left(\gamma^{2}-1\right) F_{f}^{s} F_{g}^{t}\right)^{\omega_{f} \omega_{g}}\right)^{1 / \sum_{f=1}^{h} \sum_{g=f}^{h} \omega_{f} \omega_{g}}}\right\rangle .
\end{aligned}
$$

Let

Then,

$$
\begin{aligned}
M_{f g} & =B_{f}^{s} B_{g}^{t}+\left(\gamma^{2}-1\right) A_{f}^{s} A_{g}^{t}, \\
N_{f g} & =B_{f}^{s} B_{g}^{t}-A_{f}^{s} A_{g}^{t}, \\
O_{f g} & =C_{f}^{s} C_{g}^{t}-D_{f}^{s} D_{g}^{t}, \\
P_{f g} & =C_{f}^{s} C_{g}^{t}+\left(\gamma^{2}-1\right) D_{f}^{s} D_{g}^{t}, \\
Q_{f g} & =E_{f}^{s} E_{g}^{t}-F_{f}^{s} F_{g}^{t}, \\
R_{f g} & =E_{f}^{s} E_{g}^{t}+\left(\gamma^{2}-1\right) F_{f}^{s} F_{g}^{t} .
\end{aligned}
$$

$$
\begin{aligned}
& \frac{\Theta_{f=1}^{h} \oplus_{g=f}^{h} \omega_{f} \omega_{g}\left(h \zeta_{f} \beta_{f}\right)^{s} \otimes\left(h \zeta_{g} \beta_{g}\right)^{t}}{\sum_{f=1}^{h} \sum_{g=f}^{h} \omega_{f} \omega_{g}}=\left\langle\frac{\left(\prod_{f=1}^{h} \prod_{g=f}^{h} M_{f g}^{\omega_{f} \omega_{g}}\right)^{\left(1 / \sum_{f=1}^{h} \sum_{g=f}^{h} \omega_{f} \omega_{g}\right)}-\left(\prod_{f=1}^{h} \prod_{g=f}^{h} N_{f g}^{\omega_{f} \omega_{g}}\right)^{\left(1 / \sum_{f=1}^{h} \sum_{g=f}^{h} \omega_{f} \omega_{g}\right)}}{\left(\prod_{f=1}^{h} \prod_{g=f}^{h} M_{f g}^{\omega_{f} \omega_{g}}\right)^{\left(1 / \sum_{f=1}^{h} \sum_{g=f}^{h} \omega_{f} \omega_{g}\right)}+(\gamma-1)\left(\prod_{f=1}^{h} \prod_{g=f}^{h} N_{f g}^{\omega_{f} \omega_{g}}\right)^{\left(1 / \sum_{f=1}^{h} \sum_{g=f}^{h} \omega_{f} \omega_{g}\right)}},\right. \\
& \cdot \frac{\gamma\left(\prod_{f=1}^{h} \prod_{g=f}^{h} O_{f g}^{\omega_{f} \omega_{g}}\right)\left(11 \sum_{f=1}^{h} \sum_{g=f}^{h} \omega_{f} \omega_{g}\right)}{\left(\prod_{f=1}^{h} \prod_{g=f}^{h} P_{f g}^{\omega_{f} \omega_{g}}\right)^{\left(1 / \sum_{f=1}^{h} \sum_{g=f}^{h} \omega_{f} \omega_{g}\right)}+(\gamma-1)\left(\prod_{f=1}^{h} \prod_{g=f}^{h} O_{f g}^{\omega_{f} \omega_{g}}\right)^{\left(1 / \sum_{f=1}^{h} \sum_{g=f}^{h} \omega_{f} \omega_{g}\right)}}, \\
& \left.\cdot \frac{\gamma\left(\prod_{f=1}^{h} \prod_{g=f}^{h} Q_{f g}^{\omega_{f} \omega_{g}}\right)^{\left(1 / \sum_{f=1}^{h} \sum_{g=f}^{h} \omega_{f} \omega_{g}\right)}}{\left(\prod_{f=1}^{h} \prod_{g=f}^{h} R_{f g}^{\omega_{f} \omega_{g}}\right)^{\left(1 / \sum_{f=1}^{h} \sum_{g=f}^{h} \omega_{f} \omega_{g}\right)}+(\gamma-1)\left(\prod_{f=1}^{h} \prod_{g=f}^{h} Q_{f g}^{\omega_{f} \omega_{g}}\right)^{\left(1 / \sum_{f=1}^{h} \sum_{g=f}^{h} \omega_{f} \omega_{g}\right)}}\right\rangle \cdot
\end{aligned}
$$


Thus,

$$
\begin{aligned}
& \operatorname{PFHWPIGHM}^{s, t}\left(\beta_{1}, \beta_{2}, \ldots, \beta_{h}\right)=\left(\frac{\oplus_{f=1}^{h} \oplus_{g=f}^{h} \omega_{f} \omega_{g}\left(h \zeta_{f} \beta_{f}\right)^{s} \otimes\left(h \zeta_{g} \beta_{g}\right)^{t}}{\sum_{f=1}^{h} \sum_{g=f}^{h} \omega_{f} \omega_{g}}\right)^{(1 /(s+t))}
\end{aligned}
$$

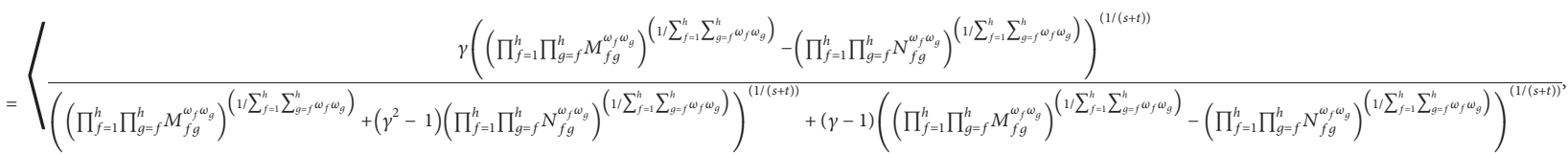

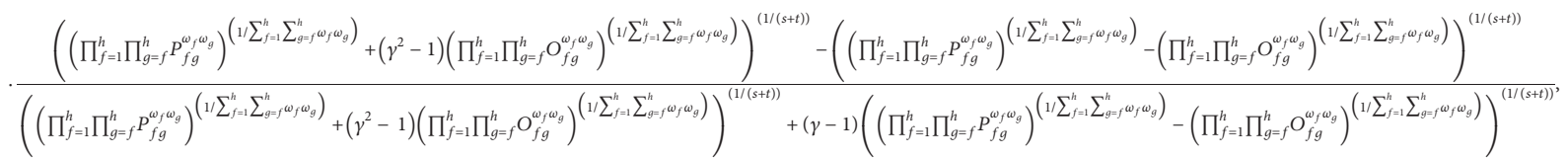

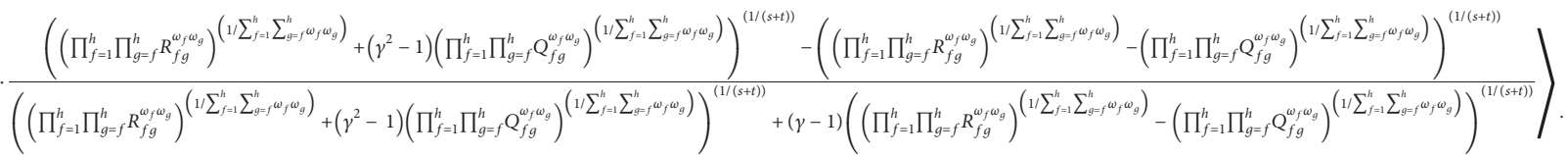

Therefore, Theorem 1 is true.

Theorem 2 (idempotency). Let $s, t \geq 0$ and $\beta_{f}=\left\langle\mu_{f}, \eta_{f}, \nu_{f}\right\rangle(f=1,2, \ldots, h)$ be multiple PFNs, and $\omega=\left(\omega_{1}, \omega_{2}, \ldots, \omega_{h}\right)^{T}$, satisfying $\omega_{f}=(1 / h)(f=1,2, \ldots, h)$ and $\sum_{g=1}^{h} \omega_{g}=1$. When $\beta_{f}=\beta(f=1,2, \ldots, h)$, the PFHWPIGHM operator has the property of idempotency.
Proof. Since $\beta_{f}=\beta$ for each $f, \sup \left(\beta_{f}, \beta_{g}\right)=1$ and $\varphi\left(\beta_{f}\right)=h-1$.

Then, $\varepsilon_{f}=\left(1+\varphi\left(\beta_{f}\right) / \sum_{e=1}^{h}\left(1+\varphi\left(\beta_{e}\right)\right)\right)=(1 / h), \quad\left(h \varepsilon_{f}\right.$ $\left.\omega_{f} / \sum_{e=1}^{h} \varepsilon_{e} \omega_{e}\right)=h \omega_{f}$, and $\left(h \varepsilon_{g} \omega_{g} / \sum_{e=1}^{h} \varepsilon_{e} \omega_{e}\right)=h \omega_{g}$.

$$
\begin{aligned}
& \operatorname{PFHWPIGHM}^{s, t}\left(\beta_{1}, \beta_{2}, \ldots, \beta_{h}\right) \\
& =\left(\frac{\oplus_{f=1}^{h} \oplus_{g=f}^{h} \omega_{f} \omega_{g}\left(\left(h \varepsilon_{f} \omega_{f} / \sum_{e=1}^{h} \varepsilon_{e} \omega_{e}\right) \beta_{f}\right)^{s} \otimes\left(h \varepsilon_{g} \omega_{g} / \sum_{e=1}^{h} \varepsilon_{e} \omega_{e} \beta_{g}\right)^{t}}{\sum_{f=1}^{h} \sum_{g=f}^{h} \omega_{f} \omega_{g}}\right)^{(1 /(s+t))} \\
& =\left(\frac{\oplus_{f=1}^{h} \oplus_{g=f}^{h} \omega_{f} \omega_{g}(\beta)^{s} \otimes(\beta)^{t}}{\sum_{f=1}^{h} \sum_{g=f}^{h} \omega_{f} \omega_{g}}\right)^{(1 /(s+t))} \\
& =\left(\beta^{s+t}\right)^{(1 /(s+t))} \\
& =\beta \text {. }
\end{aligned}
$$

Therefore, Theorem 2 is true.

Next, we will discuss some special instances of the PFHWPIGHM operator.
(1) When $\gamma=1$, the PFHWPIGHM operator is degenerated to picture fuzzy Algebraic weighted power improved generalized Heronian mean (PFAWPIGHM) operator as follows: 
$\operatorname{PFHWPIGHM}^{s, t}\left(\beta_{1}, \beta_{2}, \ldots, \beta_{h}\right)$

$$
\begin{aligned}
& =\left\langle\left(1-\left(\prod_{f=1}^{h} \prod_{g=f}^{h}\left(1-\left(1-\left(1-\mu_{f}\right)^{\left(h \varepsilon_{f} \omega_{f} / \sum_{e=1}^{h} \varepsilon_{e} \omega_{e}\right)}\right)^{s}\left(1-\left(1-\mu_{g}\right)\left(h \varepsilon_{g} \omega_{g} \sum_{e=1}^{h} \varepsilon_{e} \omega_{e}\right)\right)^{t}\right)^{\omega_{f} \omega_{g}}\right)^{\left(1 / \sum_{f=1}^{h} \sum_{g=f}^{h} \omega_{f} \omega_{g}\right)}\right)^{(1 /(s+t))},\right. \\
& 1-\left(1-\left(\prod_{f=1}^{h} \prod_{g=f}^{h}\left(1-\left(1-\eta_{f}^{\left(h \varepsilon_{f} \omega_{f} / \sum_{e=1}^{h} \varepsilon_{e} \omega_{e}\right)}\right)\right)^{s}\left(1-\eta_{g}^{\left(h \varepsilon_{g} \omega_{g} / \sum_{e=1}^{h} \varepsilon_{e} \omega_{e}\right)}\right)^{t}\right)^{\omega_{f} \omega_{g}}\right)^{\left(1 / \sum_{f=1}^{h} \sum_{g=f}^{h} \omega_{f} \omega_{g}\right)}, \\
& \left.\left.1-\left(1-\left(\prod_{f=1}^{h} \prod_{g=f}^{h}\left(1-\left(1-v_{f}^{\left(h \varepsilon_{f} \omega_{f} / \sum_{e=1}^{h} \varepsilon_{e} \omega_{e}\right)}\right)\right)^{s}\left(1-v_{g}^{\left(h \varepsilon_{g} \omega_{g} \sum_{e=1}^{h} \varepsilon_{e} \omega_{e}\right)}\right)^{t}\right)^{\omega_{f} \omega_{g}}\right)^{\left(1 / \sum_{f=1}^{h} \sum_{g=f}^{h} \omega_{f} \omega_{g}\right)}\right)^{(1 /(s+t))}\right\rangle
\end{aligned}
$$

(2) When $\gamma=2$, the PFHWPIGHM operator is degenerated to picture fuzzy Einstein weighted power improved generalized Heronian mean (PFEWPIGHM) operator as follows:

PFHWPIGHM $^{\mathrm{s}, t}\left(\beta_{1}, \beta_{2}, \ldots, \beta_{h}\right)$

$$
\begin{aligned}
& =\left\langle\frac{2\left(\left(\prod_{f=1}^{h} \prod_{g=f}^{h} M_{f g}^{\omega_{f} \omega_{g}}\right)^{\left(1 / \sum_{f=1}^{h} \sum_{g=f}^{h} \omega_{f} \omega_{g}\right)}-\left(\prod_{f=1}^{h} \prod_{g=f}^{h} N_{f g}^{\omega_{f} \omega_{g}}\right)^{\left(1 / \sum_{f=1}^{h} \sum_{g=f}^{h} \omega_{f} \omega_{g}\right)}\right)^{(1 / / s+t))}}{\left(\left(\prod_{f=1}^{h} \prod_{g=f}^{h} M_{f g}^{\omega_{f} \omega_{g}}\right)^{\left(1 / \sum_{f=1}^{h} \sum_{g=f}^{h} \omega_{f} \omega_{g}\right)}+3\left(\prod_{f=1}^{h} \prod_{g=f}^{h} N_{f g}^{\omega_{f} \omega_{g}}\right)^{\left(1 / \sum_{f=1}^{h} \sum_{g=f}^{h} \omega_{f} \omega_{g}\right)}\right)^{(1 / / s+t))}+\left(\left(\prod_{f=1}^{h} \prod_{g=f}^{h} M_{f g}^{\omega_{f} \omega_{g}}\right)^{\left(1 / \sum_{f=1}^{h} \sum_{g=f}^{h} \omega_{f} \omega_{g}\right)}-\left(\prod_{f=1}^{h} \prod_{g=f}^{h} N_{f g}^{\omega_{f} \omega_{g}}\right)^{\left(1 / \sum_{f=1}^{h} \sum_{g=f}^{h}\left(\omega_{f} \omega_{g}\right)\right.}\right)}\right)^{1 / s+t+1},
\end{aligned}
$$

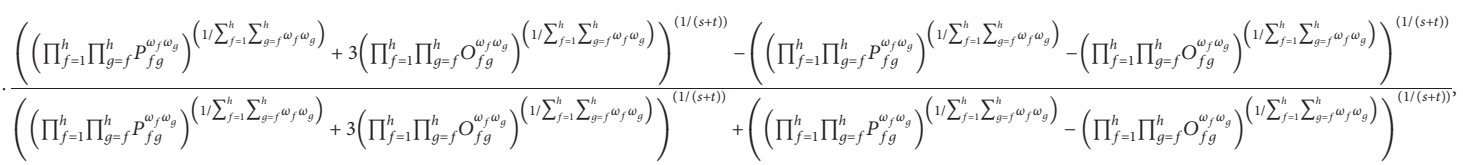

$$
\begin{aligned}
& \left.\cdot \frac{\left(\left(\prod_{f=1}^{h} \prod_{g=f}^{h} R_{f g}^{\omega_{f} \omega_{g}}\right)^{\left(1 / \sum_{f=1}^{h} \sum_{g=f}^{h} \omega_{f} \omega_{g}\right)}+3\left(\prod_{f=1}^{h} \prod_{g=f}^{h} Q_{f g}^{\omega_{f} \omega_{g}}\right)^{\left(1 / \sum_{f=1}^{h} \sum_{g=f}^{h} \omega_{f} \omega_{g}\right)}\right)^{(1 / / s+t))}-\left(\left(\prod_{f=1}^{h} \prod_{g=f}^{h} R_{f g}^{\omega_{f} \omega_{g}}\right)^{\left(1 / \sum_{f=1}^{h} \sum_{g=f}^{h} \omega_{f} \omega_{g}\right)}-\left(\prod_{f=1}^{h} \prod_{g=f}^{h} Q_{f g}^{\omega_{f} \omega_{g}}\right)^{\left(1 / \sum_{f=1}^{h} \sum_{g=f}^{h} \omega_{f} \omega_{g}\right)}\right)}{\left(\left(\prod_{f=1}^{h} \prod_{g=f}^{h} R_{f g}^{\omega_{f} \omega_{g}}\right)^{\left(1 / \sum_{f=1}^{h} \sum_{g=f}^{h} \omega_{f} \omega_{g}\right)}+3\left(\prod_{f=1}^{h} \prod_{g=f}^{h} Q_{f g}^{\omega_{f} \omega_{g}}\right)^{(1 / /(s+t))}\right.}\right\rangle .
\end{aligned}
$$


Here,

$$
\begin{aligned}
& M_{f g}=\left(\left(1+\mu_{f}\right)^{\left(h \varepsilon_{f} \omega_{f} / \sum_{e=1}^{h} \varepsilon_{e} \omega_{e}\right)}+3\left(1-\mu_{f}\right)^{\left(h \varepsilon_{f} \omega_{f} / \sum_{e=1}^{h} \varepsilon_{e} \omega_{e}\right)}\right)^{s}\left(\left(1+\mu_{g}\right)^{\left(h \varepsilon_{g} \omega_{g} / \sum_{e=1}^{h} \varepsilon_{e} \omega_{e}\right)}+3\left(1-\mu_{g}\right)^{\left(h \varepsilon_{g} \omega_{g} / \sum_{e=1}^{h} \varepsilon_{e} \omega_{e}\right)}\right)^{t} \\
& +3\left(\left(1+\mu_{f}\right)^{\left(h \varepsilon_{f} \omega_{f} / \sum_{e=1}^{h} \varepsilon_{e} \omega_{e}\right)}-\left(1-\mu_{f}\right)^{\left(h \varepsilon_{f} \omega_{f} / \sum_{e=1}^{h} \varepsilon_{e} \omega_{e}\right)}\right)^{s}\left(\left(1+\mu_{g}\right)^{\left(h \varepsilon_{g} \omega_{g} / \sum_{e=1}^{h} \varepsilon_{e} \omega_{e}\right)}-\left(1-\mu_{g}\right)^{\left(h \varepsilon_{g} \omega_{g} / \sum_{e=1}^{h} \varepsilon_{e} \omega_{e}\right)}\right)^{t},
\end{aligned}
$$

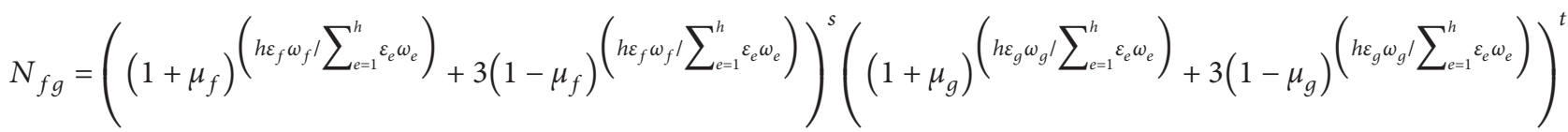

$$
\begin{aligned}
& -\left(\left(1+\mu_{f}\right)^{\left(h \varepsilon_{f} \omega_{f} / \sum_{e=1}^{h} \varepsilon_{e} \omega_{e}\right)}-\left(1-\mu_{f}\right)^{\left(h \varepsilon_{f} \omega_{f} / \sum_{e=1}^{h} \varepsilon_{e} \omega_{e}\right)}\right)^{s}\left(\left(1+\mu_{g}\right)^{\left(h \varepsilon_{g} \omega_{g} / \sum_{e=1}^{h} \varepsilon_{e} \omega_{e}\right)}-\left(1-\mu_{g}\right)^{\left(h \varepsilon_{g} \omega_{g} / \sum_{e=1}^{h} \varepsilon_{e} \omega_{e}\right)}\right)^{t}, \\
& O_{f g}=\left(\left(2-\eta_{f}\right)^{\left(h \varepsilon_{f} \omega_{f} / \sum_{e=1}^{h} \varepsilon_{e} \omega_{e}\right)}+3 \eta_{f}^{\left(h \varepsilon_{f} \omega_{f} / \sum_{e=1}^{h} \varepsilon_{e} \omega_{e}\right)}\right)^{s}\left(\left(2-\eta_{g}\right)^{\left(h \varepsilon_{g} \omega_{g} / \sum_{e=1}^{h} \varepsilon_{e} \omega_{e}\right)}+3 \eta_{g}\left(h \varepsilon_{g} \omega_{g} / \sum_{e=1}^{h} \varepsilon_{e} \omega_{e}\right)\right)^{t} \\
& -\left(\left(2-\eta_{f}\right)^{\left(h \varepsilon_{f} \omega_{f} / \sum_{e=1}^{h} \varepsilon_{e} \omega_{e}\right)}-\eta_{f}^{\left(h \varepsilon_{f} \omega_{f} / \sum_{e=1}^{h} \varepsilon_{e} \omega_{e}\right)}\right)^{s}\left(\left(2-\eta_{g}\right)^{\left(h \varepsilon_{g} \omega_{g} / \sum_{e=1}^{h} \varepsilon_{e} \omega_{e}\right)}-\eta_{g}^{\left(h \varepsilon_{g} \omega_{g} / \sum_{e=1}^{h} \varepsilon_{e} \omega_{e}\right)}\right)^{t}, \\
& P_{f g}=\left(\left(2-\eta_{f}\right)\left(h \varepsilon_{f} \omega_{f} / \sum_{e=1}^{h} \varepsilon_{e} \omega_{e}\right)+3 \eta_{f}^{\left(h \varepsilon_{f} \omega_{f} / \sum_{e=1}^{h} \varepsilon_{e} \omega_{e}\right)}\right)^{s}\left(\left(2-\eta_{g}\right)\left(h \varepsilon_{g} \omega_{g} / \sum_{e=1}^{h} \varepsilon_{e} \omega_{e}\right)+3 \eta_{g}\left(h \varepsilon_{g} \omega_{g} / \sum_{e=1}^{h} \varepsilon_{e} \omega_{e}\right)\right)^{t} \\
& +3\left(\left(2-\eta_{f}\right)\left(h \varepsilon_{f} \omega_{f} / \sum_{e=1}^{h} \varepsilon_{e} \omega_{e}\right)-\eta_{f}^{\left(h \varepsilon_{f} \omega_{f} / \sum_{e=1}^{h} \varepsilon_{e} \omega_{e}\right)}\right)^{s}\left(\left(2-\eta_{g}\right)^{\left(h \varepsilon_{g} \omega_{g} / \sum_{e=1}^{h} \varepsilon_{e} \omega_{e}\right)}-\eta_{g}^{\left(h \varepsilon_{g} \omega_{g} / \sum_{e=1}^{h} \varepsilon_{e} \omega_{e}\right)}\right)^{t},
\end{aligned}
$$

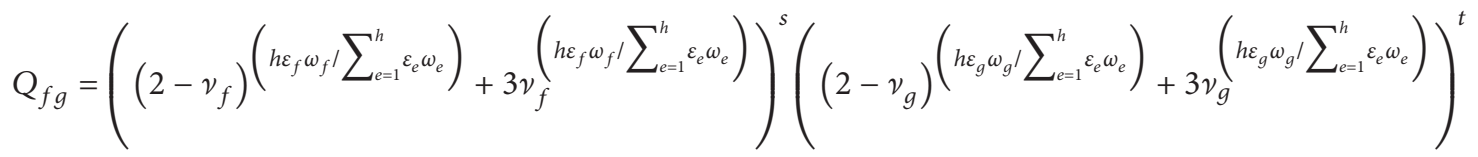

$$
\begin{aligned}
& -\left(\left(2-v_{f}\right)\left(h \varepsilon_{f} \omega_{f} / \sum_{e=1}^{h} \varepsilon_{e} \omega_{e}\right)-v_{f}^{\left(h \varepsilon_{f} \omega_{f} / \sum_{e=1}^{h} \varepsilon_{e} \omega_{e}\right)}\right)^{s}\left(\left(2-v_{g}\right)^{\left(h \varepsilon_{g} \omega_{g} / \sum_{e=1}^{h} \varepsilon_{e} \omega_{e}\right)}-v_{g}^{\left(h \varepsilon_{g} \omega_{g} / \sum_{e=1}^{h} \varepsilon_{e} \omega_{e}\right)}\right)^{t}, \\
& R_{f g}=\left(\left(2-v_{f}\right)\left(h \varepsilon_{f} \omega_{f} / \sum_{e=1}^{h} \varepsilon_{e} \omega_{e}\right)+3 v_{f}^{\left(h \varepsilon_{f} \omega_{f} / \sum_{e=1}^{h} \varepsilon_{e} \omega_{e}\right)}\right)^{s}\left(\left(2-v_{g}\right)\left(h \varepsilon_{g} \omega_{g} / \sum_{e=1}^{h} \varepsilon_{e} \omega_{e}\right)+3 v_{g}\left(h \varepsilon_{g} \omega_{g} / \sum_{e=1}^{h} \varepsilon_{e} \omega_{e}\right)\right)^{t} \\
& +3\left(\left(2-v_{f}\right)\left(h \varepsilon_{f} \omega_{f} / \sum_{e=1}^{h} \varepsilon_{e} \omega_{e}\right)-v_{f}^{\left(h \varepsilon_{f} \omega_{f} / \sum_{e=1}^{h} \varepsilon_{e} \omega_{e}\right)}\right)^{s}\left(\left(2-v_{g}\right)\left(h \varepsilon_{g} \omega_{g} / \sum_{e=1}^{h} \varepsilon_{e} \omega_{e}\right)-v_{g}^{\left(h \varepsilon_{g} \omega_{g} / \sum_{e=1}^{h} \varepsilon_{e} \omega_{e}\right)}\right)^{t} .
\end{aligned}
$$

\subsection{PFHWGPIGHM Operator}

Definition 8. Let $s, t \geq 0$ and $\beta_{f}(f=1,2, \ldots, h)$ be multiple PFNs, and the importance of PFN $\beta_{f}$ is represented by $\omega_{f}$, meeting $\omega_{f} \geq 0$ and $\sum_{f=1}^{h} \omega_{f}=1$. Then, the picture fuzzy Hamacher weighted geometric power improved generalized Heronian mean (PFHWGPIGHM) operator is denoted as 


$$
\begin{aligned}
& \operatorname{PFHWGPIGHM}^{s, t}\left(\beta_{1}, \beta_{2}, \ldots, \beta_{h}\right)
\end{aligned}
$$

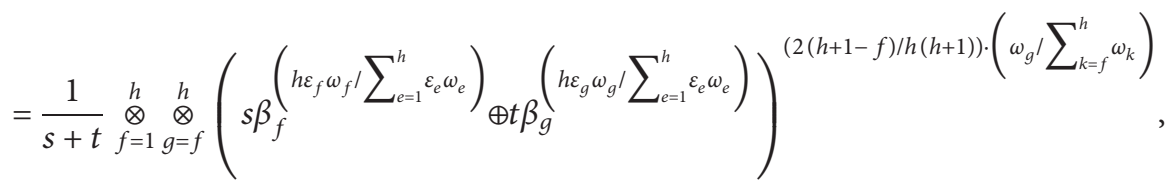

where $\varepsilon_{f}=\left(1+\varphi\left(\beta_{f}\right) / \sum_{e=1}^{h}\left(1+\varphi\left(\beta_{e}\right)\right)\right), \varphi\left(\beta_{f}\right)=\sum_{g=1, g \neq f}^{h}$ $\sup \left(\beta_{f}, \beta_{g}\right)$, and $\sup \left(\beta_{f}, \beta_{g}\right)=1-d\left(\beta_{f}, \beta_{g}\right)$.

Theorem 3. Let $s, t \geq 0$ and $\beta_{f}=\left\langle\mu_{f}, \eta_{f}, v_{f}\right\rangle(f=$ $1,2, \ldots, h)$ be multiple PFNs, and the importance of PFN $\beta_{f}$ is represented by $\omega_{f}$, meeting $\omega_{f} \geq 0$ and $\sum_{f=1}^{h} \omega_{f}=1$. Based on Hamacher operations in Definition 2, the aggregating value of PFHWGPIGHM operator in Definition 8 is still a PFN and is shown as follows:

PFHWGPIGHM $^{\mathrm{s}, t}\left(\beta_{1}, \beta_{2}, \ldots, \beta_{h}\right)$

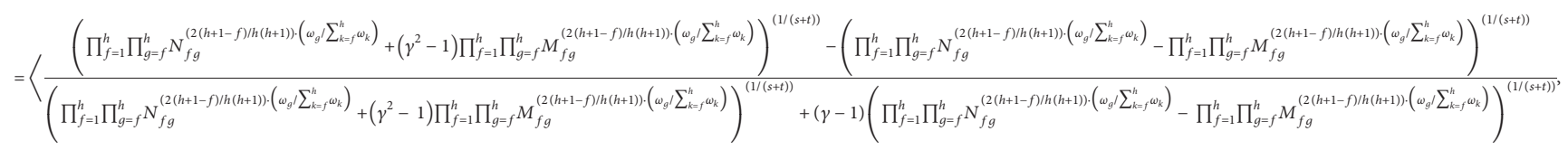

$$
\begin{aligned}
& \gamma\left(\prod_{f=1}^{h} \prod_{g=f}^{h} O_{f g}^{(2(h+1-f) / h(h+1)) \cdot\left(\omega_{g} / \sum_{k=f}^{h} \omega_{k}\right)}-\prod_{f=1}^{h} \prod_{g=f}^{h} P_{f g}^{(2(h+1-f) / h(h+1)) \cdot\left(\omega_{g} / \sum_{k=f}^{h} \omega_{k}\right)}\right)^{(1 /(s+t))}
\end{aligned}
$$

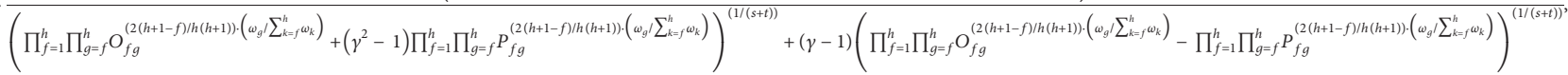

$$
\begin{aligned}
& \gamma\left(\prod_{f=1}^{h} \prod_{g=f}^{h} Q_{f g}^{(2(h+1-f) / h(h+1)) \cdot\left(\omega_{g} / \sum_{k=f}^{h} \omega_{k}\right)}-\prod_{f=1}^{h} \prod_{g=f}^{h} R_{f g}^{(2(h+1-f) / h(h+1)) \cdot\left(\omega_{g} / \sum_{k=f}^{h} \omega_{k}\right)}\right)^{(1 /(s+t))}
\end{aligned}
$$

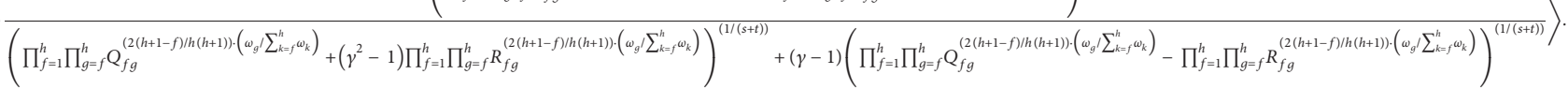

Here,

$$
\begin{aligned}
M_{f g}= & \left(\left(1+(\gamma-1)\left(1-\mu_{f}\right)\right)^{h \zeta_{f}}+\left(\gamma^{2}-1\right) \mu_{f}^{h \zeta_{f}}\right)^{s}\left(\left(1+(\gamma-1)\left(1-\mu_{g}\right)\right)^{h \zeta_{g}}+\left(\gamma^{2}-1\right) \mu_{g}^{h \zeta_{g}}\right)^{t} \\
& -\left(\left(1+(\gamma-1)\left(1-\mu_{f}\right)\right)^{h \zeta_{f}}-\mu_{f}^{h \zeta_{f}}\right)^{s}\left(\left(1+(\gamma-1)\left(1-\mu_{g}\right)\right)^{h \zeta_{g}}-\mu_{g}^{h \zeta_{g}}\right)^{t}, \\
N_{f g}= & \left(\left(1+(\gamma-1)\left(1-\mu_{f}\right)\right)^{h \zeta_{f}}+\left(\gamma^{2}-1\right) \mu_{f}^{h \zeta_{f}}\right)^{s}\left(\left(1+(\gamma-1)\left(1-\mu_{g}\right)\right)^{h \zeta_{g}}+\left(\gamma^{2}-1\right) \mu_{g}^{h \zeta_{g}}\right)^{t} \\
& +\left(\gamma^{2}-1\right)\left(\left(1+(\gamma-1)\left(1-\mu_{f}\right)\right)^{h \zeta_{f}}-\mu_{f}^{h \zeta_{f}}\right)^{s}\left(\left(1+(\gamma-1)\left(1-\mu_{g}\right)\right)^{h \zeta_{g}}-\mu_{g}^{h \zeta_{g}}\right)^{t}, \\
O_{f g}= & \left(\left(1+(\gamma-1) \eta_{f}\right)^{h \zeta_{f}}+\left(\gamma^{2}-1\right)\left(1-\eta_{f}\right)^{h \zeta_{f}}\right)^{s}\left(\left(1+(\gamma-1) \eta_{g}\right)^{h \zeta_{g}}+\left(\gamma^{2}-1\right)\left(1-\eta_{g}\right)^{h \zeta_{g}}\right)^{t} \\
& +\left(\gamma^{2}-1\right)\left(\left(1+(\gamma-1) \eta_{f}\right)^{h \zeta_{f}}-\left(1-\eta_{f}\right)^{h \zeta_{f}}\right)^{s}\left(\left(1+(\gamma-1) \eta_{g}\right)^{h \zeta_{g}}-\left(1-\eta_{g}\right)^{h \zeta_{g}}\right)^{t},
\end{aligned}
$$




$$
\begin{aligned}
& P_{f g}=\left(\left(1+(\gamma-1) \eta_{f}\right)^{h \zeta_{f}}+\left(\gamma^{2}-1\right)\left(1-\eta_{f}\right)^{h \zeta_{f}}\right)^{s}\left(\left(1+(\gamma-1) \eta_{g}\right)^{h \zeta_{g}}+\left(\gamma^{2}-1\right)\left(1-\eta_{g}\right)^{h \zeta_{g}}\right)^{t} \\
& -\left(\left(1+(\gamma-1) \eta_{f}\right)^{h \zeta_{f}}-\left(1-\eta_{f}\right)^{h \zeta_{f}}\right)^{s}\left(\left(1+(\gamma-1) \eta_{g}\right)^{h \zeta_{g}}-\left(1-\eta_{g}\right)^{h \zeta_{g}}\right)^{t} \\
& Q_{f g}=\left(\left(1+(\gamma-1) v_{f}\right)^{h \zeta_{f}}+\left(\gamma^{2}-1\right)\left(1-v_{f}\right)^{h \zeta_{f}}\right)^{s}\left(\left(1+(\gamma-1) v_{g}\right)^{h \zeta_{g}}+\left(\gamma^{2}-1\right)\left(1-v_{g}\right)^{h \zeta_{g}}\right)^{t} \\
& +\left(\gamma^{2}-1\right)\left(\left(1+(\gamma-1) v_{f}\right)^{h \zeta_{f}}-\left(1-v_{f}\right)^{h \zeta_{f}}\right)^{s}\left(\left(1+(\gamma-1) v_{g}\right)^{h \zeta_{g}}-\left(1-v_{g}\right)^{h \zeta_{g}}\right)^{t} \\
& R_{f g}=\left(\left(1+(\gamma-1) v_{f}\right)^{h \zeta_{f}}+\left(\gamma^{2}-1\right)\left(1-v_{f}\right)^{h \zeta_{f}}\right)^{s}\left(\left(1+(\gamma-1) v_{g}\right)^{h \zeta_{g}}+\left(\gamma^{2}-1\right)\left(1-v_{g}\right)^{h \zeta_{g}}\right)^{t} \\
& -\left(\left(1+(\gamma-1) v_{f}\right)^{h \zeta_{f}}-\left(1-v_{f}\right)^{h \zeta_{f}}\right)^{s}\left(\left(1+(\gamma-1) v_{g}\right)^{h \zeta_{g}}-\left(1-v_{g}\right)^{h \zeta_{g}}\right)^{t} \\
& \zeta_{f}=\frac{\varepsilon_{f} \omega_{f}}{\sum_{e=1}^{h} \varepsilon_{e} \omega_{e}}, \\
& \zeta_{g}=\frac{\varepsilon_{g} \omega_{g}}{\sum_{e=1}^{h} \varepsilon_{e} \omega_{e}} .
\end{aligned}
$$

Proof. Based on Hamacher operations of PFNs presented in

Definition 2, we get

$$
\begin{aligned}
& \beta_{f}^{h \zeta_{f}}=\left\langle\frac{\gamma \mu_{f}^{h \zeta_{f}}}{\left(1+(\gamma-1)\left(1-\mu_{f}\right)\right)^{h \zeta_{f}}+(\gamma-1) \mu_{f}^{h \zeta_{f}}}, \frac{\left(1+(\gamma-1) \eta_{f}\right)^{h \zeta_{f}}-\left(1-\eta_{f}\right)^{h \zeta_{f}}}{\left(1+(\gamma-1) \eta_{f}\right)^{h \zeta_{f}}+(\gamma-1)\left(1-\eta_{f}\right)^{h \zeta_{f}}},\right. \\
& \left.\cdot \frac{\left(1+(\gamma-1) v_{f}\right)^{h \zeta_{f}}-\left(1-v_{f}\right)^{h \zeta_{f}}}{\left(1+(\gamma-1) v_{f}\right)^{h \zeta_{f}}+(\gamma-1)\left(1-v_{f}\right)^{h \zeta_{f}}}\right\rangle \\
& \beta_{g}^{h \zeta_{g}}=\left\langle\frac{\gamma \mu_{g}^{h \zeta_{g}}}{\left(1+(\gamma-1)\left(1-\mu_{g}\right)\right)^{h \zeta_{g}}+(\gamma-1) \mu_{g}^{h \zeta_{g}}} \frac{\left(1+(\gamma-1) \eta_{g}\right)^{h \zeta_{g}}-\left(1-\eta_{f}\right)^{h \zeta_{g}}}{\left(1+(\gamma-1) \eta_{g}\right)^{h \zeta_{g}}+(\gamma-1)\left(1-\eta_{g}\right)^{h \zeta_{g}}},\right. \\
& \left.\cdot \frac{\left(1+(\gamma-1) v_{g}\right)^{h \zeta_{g}}-\left(1-v_{g}\right)^{h \zeta_{g}}}{\left(1+(\gamma-1) v_{g}\right)^{h \zeta_{g}}+(\gamma-1)\left(1-v_{g}\right)^{h \zeta_{g}}}\right\rangle \\
& s \beta_{f}^{h \zeta_{f}}=\left\langle\frac{\left(\left(1+(\gamma-1)\left(1-\mu_{f}\right)\right)^{h \zeta_{f}}+\left(\gamma^{2}-1\right) \mu_{f}^{h \zeta_{f}}\right)^{s}-\left(\left(1+(\gamma-1)\left(1-\mu_{f}\right)\right)^{h \zeta_{f}}-\mu_{f}^{h \zeta_{f}}\right)^{s}}{\left(\left(1+(\gamma-1)\left(1-\mu_{f}\right)\right)^{h \zeta_{f}}+\left(\gamma^{2}-1\right) \mu_{f}^{h \zeta_{f}}\right)^{s}+(\gamma-1)\left(\left(1+(\gamma-1)\left(1-\mu_{f}\right)\right)^{h \zeta_{f}}-\mu_{f}^{h \zeta_{f}}\right)^{s}},\right. \\
& \gamma\left(\left(1+(\gamma-1) \eta_{f}\right)^{h \zeta_{f}}-\left(1-\eta_{f}\right)^{h \zeta_{f}}\right)^{s} \\
& \cdot \overline{\left(\left(1+(\gamma-1) \eta_{f}\right)^{h \zeta_{f}}+\left(\gamma^{2}-1\right)\left(1-\eta_{f}\right)^{h \zeta_{f}}\right)^{s}+(\gamma-1)\left(\left(1+(\gamma-1) \eta_{f}\right)^{h \zeta_{f}}-\left(1-\eta_{f}\right)^{h \zeta_{f}}\right)^{s}} \\
& \left.\cdot \frac{\gamma\left(\left(1+(\gamma-1) v_{f}\right)^{h \zeta_{f}}-\left(1-v_{f}\right)^{h \zeta_{f}}\right)^{s}}{\left(\left(1+(\gamma-1) v_{f}\right)^{h \zeta_{f}}+\left(\gamma^{2}-1\right)\left(1-v_{f}\right)^{h \zeta_{f}}\right)^{s}+(\gamma-1)\left(\left(1+(\gamma-1) v_{f}\right)^{h \zeta_{f}}-\left(1-v_{f}\right)^{h \zeta_{f}}\right)^{s}}\right\rangle,
\end{aligned}
$$


14

Complexity

$$
\begin{aligned}
t \beta_{g}^{h \zeta_{g}}= & \left\langle\frac{\left(\left(1+(\gamma-1)\left(1-\mu_{g}\right)\right)^{h \zeta_{g}}+\left(\gamma^{2}-1\right) \mu_{g}^{h \zeta_{g}}\right)^{t}-\left(\left(1+(\gamma-1)\left(1-\mu_{g}\right)\right)^{h \zeta_{g}}-\mu_{g}^{h \zeta_{g}}\right)^{t}}{\left(\left(1+(\gamma-1)\left(1-\mu_{g}\right)\right)^{h \zeta_{g}}+\left(\gamma^{2}-1\right) \mu_{g}^{h \zeta_{g}}\right)^{t}+(\gamma-1)\left(\left(1+(\gamma-1)\left(1-\mu_{g}\right)\right)^{h \zeta_{g}}-\mu_{g}^{h \zeta_{g}}\right)^{t}},\right. \\
& \frac{\gamma\left(\left(1+(\gamma-1) \eta_{g}\right)^{h \zeta_{g}}-\left(1-\eta_{g}\right)^{h \zeta_{g}}\right)^{t}}{\left(\left(1+(\gamma-1) \eta_{g}\right)^{h \zeta_{g}}+\left(\gamma^{2}-1\right)\left(1-\eta_{g}\right)^{h \zeta_{g}}\right)^{t}+(\gamma-1)\left(\left(1+(\gamma-1) \eta_{g}\right)^{h \zeta_{g}}-\left(1-\eta_{g}\right)^{h \zeta_{g}}\right)^{t}}, \\
& \left.\cdot \frac{\gamma\left(\left(1+(\gamma-1) v_{g}\right)^{h \zeta_{g}}-\left(1-v_{f}\right)^{h \zeta_{g}}\right)^{t}}{\left(\left(1+(\gamma-1) v_{g}\right)^{h \zeta_{g}}+\left(\gamma^{2}-1\right)\left(1-v_{g}\right)^{h \zeta_{g}}\right)^{t}+(\gamma-1)\left(\left(1+(\gamma-1) v_{g}\right)^{h \zeta_{g}}-\left(1-v_{g}\right)^{h \zeta_{g}}\right)^{t}}\right\rangle .
\end{aligned}
$$

Let

$$
\begin{aligned}
& A_{f}=\left(1+(\gamma-1)\left(1-\mu_{f}\right)\right)^{h \zeta_{f}}+\left(\gamma^{2}-1\right) \mu_{f}^{h \zeta_{f}}, \\
& B_{f}=\left(1+(\gamma-1)\left(1-\mu_{f}\right)\right)^{h \zeta_{f}}-\mu_{f}^{h \zeta_{f}}, \\
& C_{f}=\left(1+(\gamma-1) \eta_{f}\right)^{h \zeta_{f}}-\left(1-\eta_{f}\right)^{h \zeta_{f}}, \\
& D_{f}=\left(1+(\gamma-1) \eta_{f}\right)^{h \zeta_{f}}+\left(\gamma^{2}-1\right)\left(1-\eta_{f}\right)^{h \zeta_{f}}, \\
& E_{f}=\left(1+(\gamma-1) \nu_{f}\right)^{h \zeta_{f}}-\left(1-\nu_{f}\right)^{h \zeta_{f}}, \\
& F_{f}=\left(1+(\gamma-1) \nu_{f}\right)^{h \zeta_{f}}+\left(\gamma^{2}-1\right)\left(1-\nu_{f}\right)^{h \zeta_{f}}, \\
& A_{g}=\left(1+(\gamma-1)\left(1-\mu_{g}\right)\right)^{h \zeta_{g}}+\left(\gamma^{2}-1\right) \mu_{g}^{h \zeta_{g}}, \\
& B_{g}=\left(1+(\gamma-1)\left(1-\mu_{g}\right)\right)^{h \zeta_{g}}-\mu_{g}^{h \zeta_{g}}, \\
& C_{g}=\left(1+(\gamma-1) \eta_{g}\right)^{h \zeta_{g}}-\left(1-\eta_{g}\right)^{h \zeta_{g}}, \\
& D_{g}=\left(1+(\gamma-1) \eta_{g}\right)^{h \zeta_{g}}+\left(\gamma^{2}-1\right)\left(1-\eta_{g}\right)^{h \zeta_{g}}, \\
& E_{g}=\left(1+(\gamma-1) \nu_{g}\right)^{h \zeta_{g}}-\left(1-\nu_{g}\right)^{h \zeta_{g}}, \\
& F_{g}=\left(1+(\gamma-1) \nu_{g}\right)^{h \zeta_{g}}+\left(\gamma^{2}-1\right)\left(1-v_{g}\right)^{h \zeta_{g}} .
\end{aligned}
$$

Then,

$$
\begin{aligned}
& s \beta_{f}^{h \zeta_{f}} \oplus t \beta_{g}^{h \zeta_{g}}=\left\langle\frac{A_{f}^{s} A_{g}^{t}-B_{f}^{s} B_{g}^{t}}{A_{f}^{s} A_{g}^{t}+(\gamma-1) B_{f}^{s} B_{g}^{t}}, \frac{\gamma C_{f}^{s} C_{g}^{t}}{D_{f}^{s} D_{g}^{t}+(\gamma-1) C_{f}^{s} C_{g}^{t}}, \frac{\gamma E_{f}^{s} E_{g}^{t}}{F_{f}^{s} F_{g}^{t}+(\gamma-1) E_{f}^{s} E_{g}^{t}}\right\rangle, \\
& \cdot\left(s \beta_{f}^{h \zeta_{f}} \oplus t \beta_{g}^{h \zeta_{g}}\right)^{(2(h+1-f) / h(h+1)) \cdot\left(\omega_{g} / \sum_{k=f}^{h} \omega_{k}\right)}
\end{aligned}
$$




$$
\begin{aligned}
& =\left\langle\frac{\gamma\left(A_{f}^{s} A_{g}^{t}-B_{f}^{s} B_{g}^{t}\right)^{(2(h+1-f) / h(h+1)) \cdot\left(\omega_{g} / \sum_{k=f}^{h} \omega_{k}\right)}}{\left(A_{f}^{s} A_{g}^{t}+(\gamma-1) B_{f}^{s} B_{g}^{t}\right)^{(2(h+1-f) / h(h+1)) \cdot\left(\omega_{g} / \sum_{k=f}^{h} \omega_{k}\right)}+(\gamma-1)\left(A_{f}^{s} A_{g}^{t}-B_{f}^{s} B_{g}^{t}\right)^{(2(h+1-f) / h(h+1)) \cdot\left(\omega_{g} / \sum_{k=f}^{h} \omega_{k}\right)}},\right. \\
& \left(D_{f}^{s} D_{g}^{t}+(\gamma-1) C_{f}^{s} C_{g}^{t}\right)^{(2(h+1-f) / h(h+1)) \cdot\left(\omega_{g} / \sum_{k=f}^{h} \omega_{k}\right)}-\left(D_{f}^{s} D_{g}^{t}-C_{f}^{s} C_{g}^{t}\right)^{(2(h+1-f) / h(h+1)) \cdot\left(\omega_{g} / \sum_{k=f}^{h} \omega_{k}\right)} \\
& \left(D_{f}^{s} D_{g}^{t}+(\gamma-1) C_{f}^{s} C_{g}^{t}\right)^{(2(h+1-f) / h(h+1)) \cdot\left(\omega_{g} / \sum_{k=f}^{h} \omega_{k}\right)}+(\gamma-1)\left(D_{f}^{s} D_{g}^{t}-C_{f}^{s} C_{g}^{t}\right)^{(2(h+1-f) / h(h+1)) \cdot\left(\omega_{g} / \sum_{k=f}^{h} \omega_{k}\right)} \\
& \left.\frac{\left(F_{f}^{s} F_{g}^{t}+(\gamma-1) E_{f}^{s} E_{g}^{t}\right)^{(2(h+1-f) / h(h+1)) \cdot\left(\omega_{g} \sum_{k=f}^{h} \omega_{k}\right)}-\left(F_{f}^{s} F_{g}^{t}-E_{f}^{s} E_{g}^{t}\right)^{(2(h+1-f) / h(h+1)) \cdot\left(\omega_{g} / \sum_{k=f}^{h} \omega_{k}\right)}}{\left(F_{f}^{s} F_{g}^{t}+(\gamma-1) E_{f}^{s} E_{g}^{t}\right)^{(2(h+1-f) / h(h+1)) \cdot\left(\omega_{g} / \sum_{k=f}^{h} \omega_{k}\right)}+(\gamma-1)\left(F_{f}^{s} F_{g}^{t}-E_{f}^{s} E_{g}^{t}\right)^{(2(h+1-f) / h(h+1)) \cdot\left(\omega_{g} / \sum_{k=f}^{h} \omega_{k}\right)}}\right\rangle,
\end{aligned}
$$

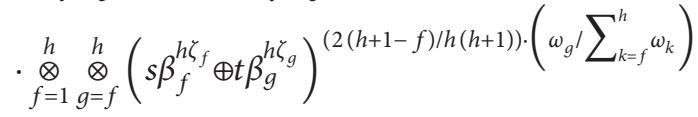

$$
\begin{aligned}
& =\left\langle\frac{\gamma \prod_{f=1}^{h} \prod_{g=f}^{h}\left(A_{f}^{s} A_{g}^{t}-B_{f}^{s} B_{g}^{t}\right)^{(2(h+1-f) / h(h+1)) \cdot\left(\omega_{g} / \sum_{k=f}^{h} \omega_{k}\right)}}{\prod_{f=1}^{h} \prod_{g=f}^{h}\left(A_{f}^{s} A_{g}^{t}+(\gamma-1) B_{f}^{s} B_{g}^{t}\right)^{(2(h+1-f) / h(h+1)) \cdot\left(\omega_{g} / \sum_{k=f}^{h} \omega_{k}\right)}+(\gamma-1) \prod_{f=1}^{h} \prod_{g=f}^{h}\left(A_{f}^{s} A_{g}^{t}-B_{f}^{s} B_{g}^{t}\right)^{(2(h+1-f) / h(h+1)) \cdot\left(\omega_{g} / \sum_{k=f}^{h} \omega_{k}\right)}},\right. \\
& . \prod_{f=1}^{h} \prod_{g=f}^{h}\left(D_{f}^{s} D_{g}^{t}+(\gamma-1) C_{f}^{s} C_{g}^{t}\right)^{(2(h+1-f) / h(h+1)) \cdot\left(\omega_{g} / \sum_{k=f}^{h} \omega_{k}\right)}-\prod_{f=1}^{h} \prod_{g=f}^{h}\left(D_{f}^{s} D_{g}^{t}-C_{f}^{s} C_{g}^{t}\right)^{(2(h+1-f) / h(h+1)) \cdot\left(\omega_{g} / \sum_{k=f}^{h} \omega_{k}\right)} \\
& \prod_{f=1}^{h} \prod_{g=f}^{h}\left(D_{f}^{s} D_{g}^{t}+(\gamma-1) C_{f}^{s} C_{g}^{t}\right)^{(2(h+1-f) / h(h+1)) \cdot\left(\omega_{g} / \sum_{k=f}^{h} \omega_{k}\right)}+(\gamma-1) \prod_{f=1}^{h} \prod_{g=f}^{h}\left(D_{f}^{s} D_{g}^{t}-C_{f}^{s} C_{g}^{t}\right)^{(2(h+1-f) / h(h+1)) \cdot\left(\omega_{g} / \sum_{k=f}^{h} \omega_{k}\right)}, \\
& \cdot \frac{\prod_{f=1}^{h} \prod_{g=f}^{h}\left(F_{f}^{s} F_{g}^{t}+(\gamma-1) E_{f}^{s} E_{g}^{t}\right)^{(2(h+1-f) / h(h+1)) \cdot\left(\omega_{g} / \sum_{k=f}^{h} \omega_{k}\right)}-\prod_{f=1}^{h} \prod_{g=f}^{h}\left(F_{f}^{s} F_{g}^{t}-E_{f}^{s} E_{g}^{t}\right)^{(2(h+1-f) / h(h+1)) \cdot\left(\omega_{g} / \sum_{k=f}^{h} \omega_{k}\right)}}{\left.\prod_{f=1}^{h} \prod_{g=f}^{h}\left(F_{f}^{s} F_{g}^{t}+(\gamma-1) E_{f}^{s} E_{g}^{t}\right)^{(2(h+1-f) / h(h+1)) \cdot\left(\omega_{g} / \sum_{k=f}^{h} \omega_{k}\right)}+(\gamma-1) \prod_{f=1}^{h} \prod_{g=f}^{h}\left(F_{f}^{s} F_{g}^{t}-E_{f}^{s} E_{g}^{t}\right)^{(2(h+1-f) / h(h+1)) \cdot\left(\omega_{g} / \sum_{k=f}^{h} \omega_{k}\right)}\right\rangle .}
\end{aligned}
$$

Let

Thus,

$$
\begin{aligned}
M_{f g} & =A_{f}^{s} A_{g}^{t}-B_{f}^{s} B_{g}^{t}, \\
N_{f g} & =A_{f}^{s} A_{g}^{t}+(\gamma-1) B_{f}^{s} B_{g}^{t}, \\
O_{f g} & =D_{f}^{s} D_{g}^{t}+(\gamma-1) C_{f}^{s} C_{g}^{t}, \\
P_{f g} & =D_{f}^{s} D_{g}^{t}-C_{f}^{s} C_{g}^{t}, \\
Q_{f g} & =F_{f}^{s} F_{g}^{t}+(\gamma-1) E_{f}^{s} E_{g}^{t}, \\
R_{f g} & =F_{f}^{s} F_{g}^{t}-E_{f}^{s} E_{g}^{t} .
\end{aligned}
$$


$\operatorname{PFHWGPIGHM}^{s, t}\left(\beta_{1}, \beta_{2}, \ldots, \beta_{h}\right)=\frac{1}{s+t}{\underset{f=1}{\otimes}}_{g=f}^{h}\left(s \beta_{f}^{h}\left(h \varepsilon_{f} \omega_{f} / \sum_{c=1}^{h} \varepsilon_{e} \omega_{e}\right)_{\oplus t \beta_{g}}^{\left(h k_{g} \omega_{g} / \sum_{e=1}^{h} \varepsilon_{e} \omega_{e}\right)}\right)^{(2(h+1-f) h(h+1)) \cdot\left(\omega_{g} / \sum_{k=f}^{h} \omega_{k}\right)}$

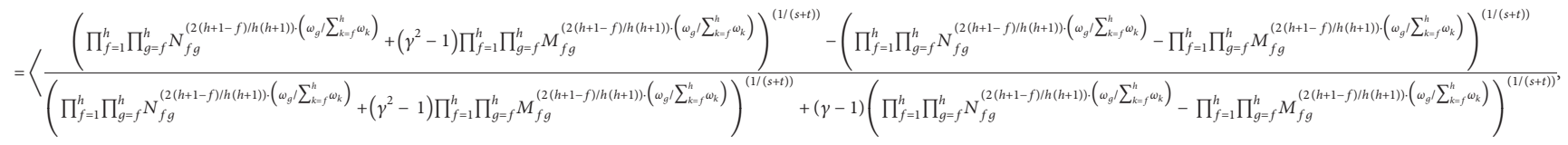

$$
\begin{aligned}
& \gamma\left(\prod_{f=1}^{h} \prod_{g=f}^{h} O_{f g}^{(2(h+1-f) / h(h+1)) \cdot\left(\omega_{g} / \sum_{k=f}^{h} \omega_{k}\right)}-\prod_{f=1}^{h} \prod_{g=f}^{h} P_{f g}^{(2(h+1-f) / h(h+1)) \cdot\left(\omega_{g} \sum_{k=f}^{h} \omega_{k}\right)}\right)^{(1 /(s+t))}
\end{aligned}
$$

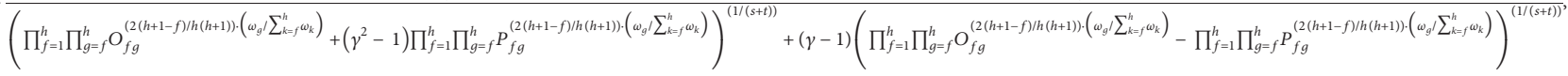

$$
\begin{aligned}
& \gamma\left(\prod_{f=1}^{h} \prod_{g=f}^{h} Q_{f g}^{(2(h+1-f) / h(h+1)) \cdot\left(\omega_{g} \sum_{k=\omega^{\prime}}^{h}\right)}-\prod_{f=1}^{h} \prod_{g=f}^{h} R_{f g}^{(2(h+1-f) / h(h+1)) \cdot\left(\omega_{g} \sum_{k=f}^{h} \omega_{k}\right)}\right)^{(1 /(s+t))}
\end{aligned}
$$

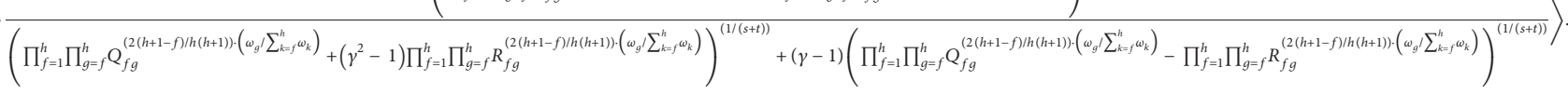

Therefore, Theorem 3 is true.

Proof. Since $\beta_{f}=\beta$ for each $f, \sup \left(\beta_{f}, \beta_{g}\right)=1$, and

Theorem 4 (idempotency). Let $s, t \geq 0$ and $\beta_{f}=\left\langle\mu_{f}, \eta_{f}, v_{f}\right\rangle(f=1,2, \ldots, h)$ be multiple PFNs, and $\omega=\left(\omega_{1}, \omega_{2}, \ldots, \omega_{h}\right)^{T}$, satisfying $\omega_{f}=(1 / n)(f=1,2, \ldots, h)$ and $\sum_{g=1}^{h} \omega_{g}=1$. When $\beta_{f}=\beta(f=1,2, \ldots, h)$, the $\varphi\left(\beta_{f}\right)=h-1$, Then, $\varepsilon_{f}=\left(1+\varphi\left(\beta_{f}\right) / \sum_{e=1}^{h}\left(1+\varphi\left(\beta_{e}\right)\right)\right)=$ $(1 / h)$, and $\quad\left(h \varepsilon_{f} \omega_{f} / \sum_{e=1}^{h} \varepsilon_{e} \omega_{e}\right)=h \omega_{f}=1 . \quad\left(h \varepsilon_{g} \omega_{g} /\right.$ $\left.\sum_{e=1}^{h} \varepsilon_{e} \omega_{e}\right)=h \omega_{g}=1$.

Thus,

$$
\begin{aligned}
& \text { PFHWGPIGHM }^{s, t}\left(\beta_{1}, \beta_{2}, \ldots, \beta_{h}\right)
\end{aligned}
$$

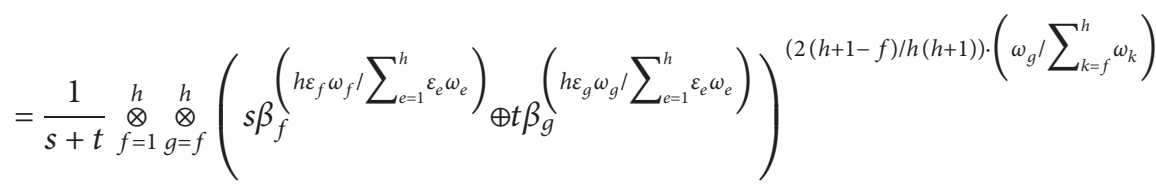

$$
\begin{aligned}
& =\frac{1}{s+t} \stackrel{h}{\otimes} \stackrel{h}{\otimes}(s \beta \oplus t \beta)^{(2(h+1-f) / h(h+1)) \cdot(1 / h+1-f)} \\
& =\frac{1}{s+t}\left((s \beta \oplus t \beta)^{h(h+1) / 2}\right)^{(2 / h(h+1))} \\
& =\beta \text {. }
\end{aligned}
$$

Therefore, Theorem 4 is true.

Next, we will discuss some special instances of the PFHWGPIGHM operator.
(1) When $\gamma=1$, the PFHWGPIGHM operator is degenerated to picture fuzzy Algebraic weighted geometric power improved generalized Heronian mean (PFAWGPIGHM) operator as follows: 


$$
\begin{aligned}
& \operatorname{PFHWGPIGHM}^{s, t}\left(\beta_{1}, \beta_{2}, \ldots, \beta_{h}\right)=
\end{aligned}
$$

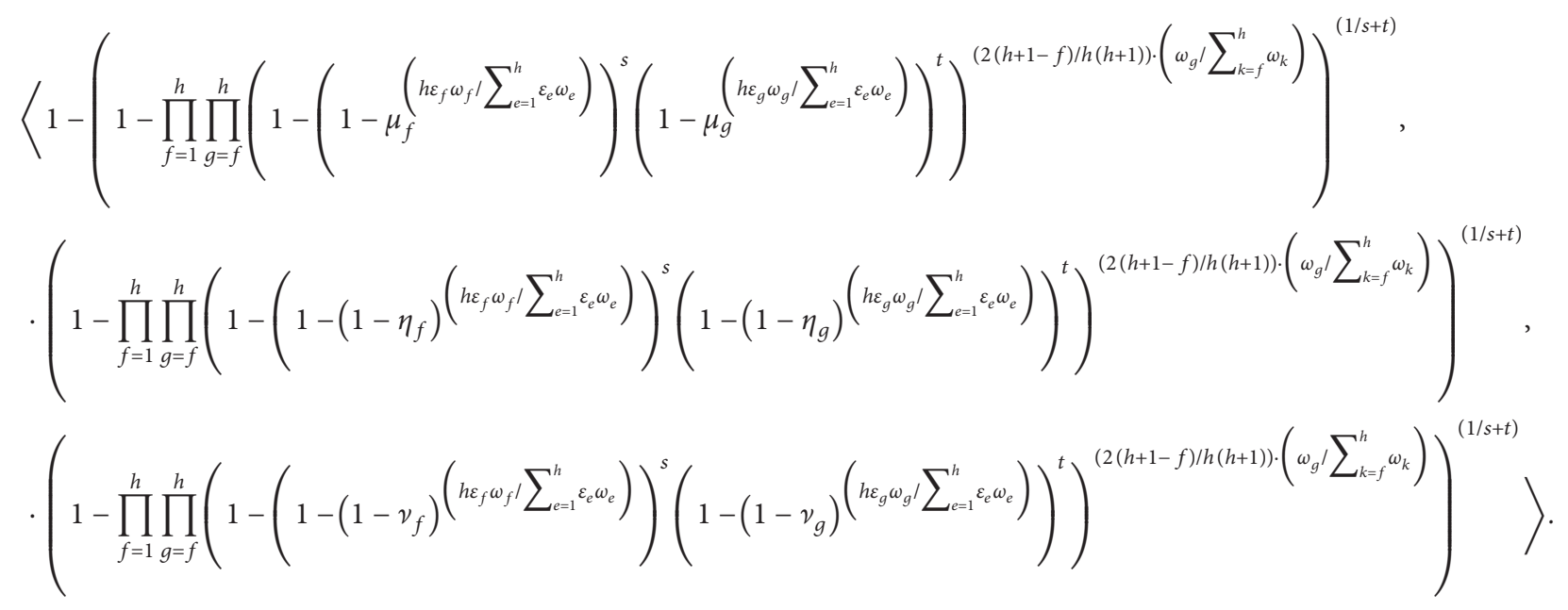

(2) When $\gamma=2$, the PFHWGPIGHM operator is degenerated to picture fuzzy Einstein weighted geometric power improved generalized Heronian mean (PFEWGPIGHM) operator as follows:

$\operatorname{PFEWGPIGHM}^{\mathrm{s}, t}\left(\beta_{1}, \beta_{2}, \ldots, \beta_{h}\right)=$

$$
\begin{aligned}
& \cdot\left\langle\frac{\left.\left(\prod_{f=1}^{h} \prod_{g=f}^{h} N_{f g}^{(2(h+1-f) / h(h+1)) \cdot\left(\omega_{g} / \sum_{k=f}^{h} \omega_{k}\right)}+3 \prod_{f=1}^{h} \prod_{g=f}^{h} M_{f g}^{(2(h+1-f) / h(h+1)) \cdot\left(\omega_{g} / \sum_{k=f}^{h} \omega_{k}\right)}\right)^{(1 /(s+t))}-\left(\prod_{f=1}^{h} \prod_{g=f}^{h} N_{f g}^{(2(h+1-f) / h(h+1)) \cdot\left(\omega_{g} / \sum_{k=f}^{h} \omega_{k}\right)}-\prod_{f=1}^{h} \prod_{g=f}^{h} M_{f g}^{(2(h+1-f) / h(h+1)) \cdot\left(\omega_{g} / \sum_{k=f}^{h} \omega_{k}\right)}\right)\right)^{(1 / /(s+t))}}{\left(\prod_{f=1}^{h} \prod_{g=f}^{h} N_{f g}^{(2(h+1-f)) h(h+1)) \cdot\left(\omega_{g} / \sum_{k=f}^{h} \omega_{k}\right)}+3 \prod_{f=1}^{h} \prod_{g=f}^{h} M_{f g}^{(2(h+1-f) / h(h+1)) \cdot\left(\omega_{g} / \sum_{k=f}^{h} \omega_{k}\right)}\right)^{(1 /(s+t))}+\left(\prod_{f=1}^{h} \prod_{g=f}^{h} N_{f g}^{(2(h+1-f) / h(h+1)) \cdot\left(\omega_{g} / \sum_{k=f}^{h}\left(\omega_{k}\right)\right.}-\prod_{f=1}^{h} \prod_{g=f}^{h} M_{f g}^{(2(h+1-f) / h(h+1)) \cdot\left(\omega_{g} / \sum_{k=f}^{h} \omega_{k}\right)}\right)}\right. \\
& 2\left(\prod_{f=1}^{h} \prod_{g=f}^{h} O_{f g}^{(2(h+1-f) / h(h+1)) \cdot\left(\omega_{g} / \sum_{k=f}^{h} \omega_{k}\right)}-\prod_{f=1}^{h} \prod_{g=f}^{h} P_{f g}^{(2(h+1-f) / h(h+1)) \cdot\left(\omega_{g} / \sum_{k=f}^{h} \omega_{k}\right)}\right)^{(1 /(s+t))}
\end{aligned}
$$

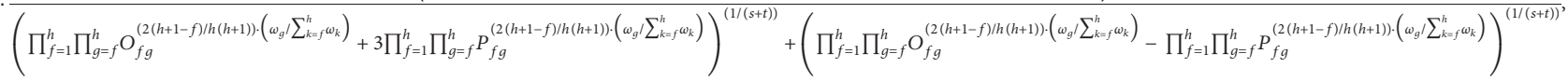

$$
\begin{aligned}
& 2\left(\prod_{f=1}^{h} \prod_{g=f}^{h} Q_{f g}^{(2(h+1-f) / h(h+1)) \cdot\left(\omega_{g} / \sum_{k=f}^{h} \omega_{k}\right)}-\prod_{f=1}^{h} \prod_{g=f}^{h} R_{f g}^{(2(h+1-f) / h(h+1)) \cdot\left(\omega_{g} / \sum_{k=f}^{h} \omega_{k}\right)}\right)^{(1 /(s+t))}
\end{aligned}
$$

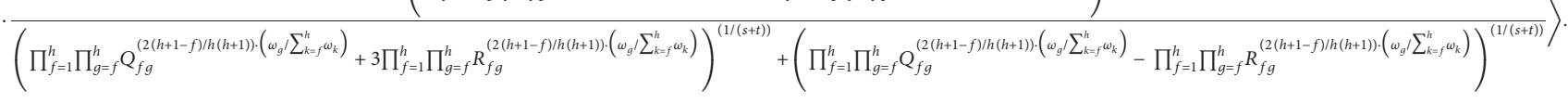


Here,

$$
\begin{aligned}
& M_{f g}=\left(\left(2-\mu_{f}\right)^{\left(h \varepsilon_{f} \omega_{f} / \sum_{e=1}^{h} \varepsilon_{e} \omega_{e}\right)}+3 \mu_{f}^{\left(h \varepsilon_{f} \omega_{f} / \sum_{e=1}^{h} \varepsilon_{e} \omega_{e}\right)}\right)^{s}\left(\left(2-\mu_{g}\right)^{\left(h \varepsilon_{g} \omega_{g} / \sum_{e=1}^{h} \varepsilon_{e} \omega_{e}\right)}+3 \mu_{g}^{\left(h \varepsilon_{g} \omega_{g} / \sum_{e=1}^{h} \varepsilon_{e} \omega_{e}\right)}\right)^{t} \\
& -\left(\left(2-\mu_{f}\right)^{\left(h \varepsilon_{f} \omega_{f} / \sum_{e=1}^{h} \varepsilon_{e} \omega_{e}\right)}-\mu_{f}^{\left(h \varepsilon_{f} \omega_{f} / \sum_{e=1}^{h} \varepsilon_{e} \omega_{e}\right)}\right)^{s}\left(\left(2-\mu_{g}\right)^{\left(h \varepsilon_{g} \omega_{g} / \sum_{e=1}^{h} \varepsilon_{e} \omega_{e}\right)}-\mu_{g}^{\left(h \varepsilon_{g} \omega_{g} / \sum_{e=1}^{h} \varepsilon_{e} \omega_{e}\right)}\right)^{t}, \\
& N_{f g}=\left(\left(2-\mu_{f}\right)\left(h \varepsilon_{f} \omega_{f} / \sum_{e=1}^{h} \varepsilon_{e} \omega_{e}\right)+3 \mu_{f}^{\left(h \varepsilon_{f} \omega_{f} / \sum_{e=1}^{h} \varepsilon_{e} \omega_{e}\right)}\right)^{s}\left(\left(2-\mu_{g}\right)^{\left(h \varepsilon_{g} \omega_{g} / \sum_{e=1}^{h} \varepsilon_{e} \omega_{e}\right)}+3 \mu_{g}\left(h \varepsilon_{g} \omega_{g} / \sum_{e=1}^{h} \varepsilon_{e} \omega_{e}\right)\right)^{t} \\
& +3\left(\left(2-\mu_{f}\right)\left(h \varepsilon_{f} \omega_{f} / \sum_{e=1}^{h} \varepsilon_{e} \omega_{e}\right)-\mu_{f}^{\left(h \varepsilon_{f} \omega_{f} / \sum_{e=1}^{h} \varepsilon_{e} \omega_{e}\right)}\right)^{s}\left(\left(2-\mu_{g}\right)\left(h \varepsilon_{g} \omega_{g} / \sum_{e=1}^{h} \varepsilon_{e} \omega_{e}\right)-\mu_{g}\left(h \varepsilon_{g} \omega_{g} / \sum_{e=1}^{h} \varepsilon_{e} \omega_{e}\right)\right)^{t}, \\
& O_{f g}=\left(\left(1+\eta_{f}\right)^{\left(h \varepsilon_{f} \omega_{f} / \sum_{e=1}^{h} \varepsilon_{e} \omega_{e}\right)}+3\left(1-\eta_{f}\right)^{\left(h \varepsilon_{f} \omega_{f} / \sum_{e=1}^{h} \varepsilon_{e} \omega_{e}\right)}\right)^{s}\left(\left(1+\eta_{g}\right)^{\left(h \varepsilon_{g} \omega_{g} / \sum_{e=1}^{h} \varepsilon_{e} \omega_{e}\right)}+3\left(1-\eta_{g}\right)\left(h \varepsilon_{g} \omega_{g} / \sum_{e=1}^{h} \varepsilon_{e} \omega_{e}\right)\right)^{t}
\end{aligned}
$$

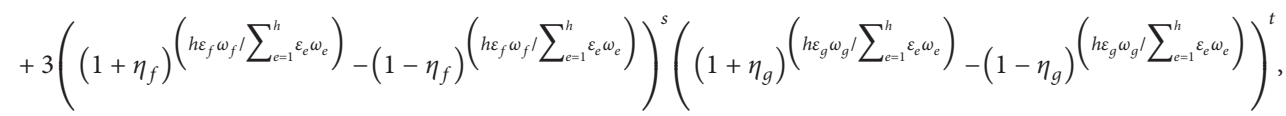

$$
\begin{aligned}
& P_{f g}=\left(\left(1+\eta_{f}\right)^{\left(h \varepsilon_{f} \omega_{f} / \sum_{e=1}^{h} \varepsilon_{e} \omega_{e}\right)}+3\left(1-\eta_{f}\right)^{\left(h \varepsilon_{f} \omega_{f} / \sum_{e=1}^{h} \varepsilon_{e} \omega_{e}\right)}\right)^{s}\left(\left(1+\eta_{g}\right)^{\left(h \varepsilon_{g} \omega_{g} / \sum_{e=1}^{h} \varepsilon_{e} \omega_{e}\right)}+3\left(1-\eta_{g}\right)^{\left(h \varepsilon_{g} \omega_{g} / \sum_{e=1}^{h} \varepsilon_{e} \omega_{e}\right)}\right)^{t} \\
& -\left(\left(1+\eta_{f}\right)^{\left(h \varepsilon_{f} \omega_{f} / \sum_{e=1}^{h} \varepsilon_{e} \omega_{e}\right)}-\left(1-\eta_{f}\right)\left(h \varepsilon_{f} \omega_{f} / \sum_{e=1}^{h} \varepsilon_{e} \omega_{e}\right)\right)^{s}\left(\left(1+\eta_{g}\right)^{\left(h \varepsilon_{g} \omega_{g} / \sum_{e=1}^{h} \varepsilon_{e} \omega_{e}\right)}-\left(1-\eta_{g}\right)^{\left(h \varepsilon_{g} \omega_{g} / \sum_{e=1}^{h} \varepsilon_{e} \omega_{e}\right)}\right)^{t},
\end{aligned}
$$

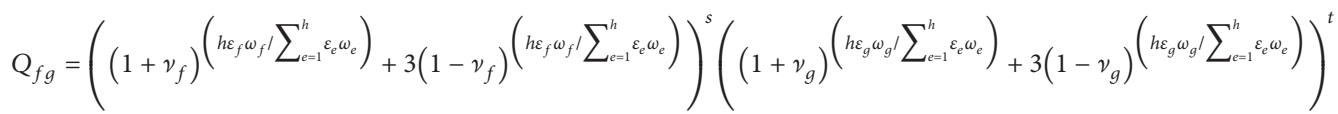

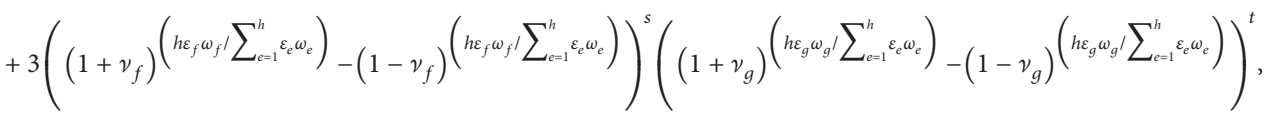

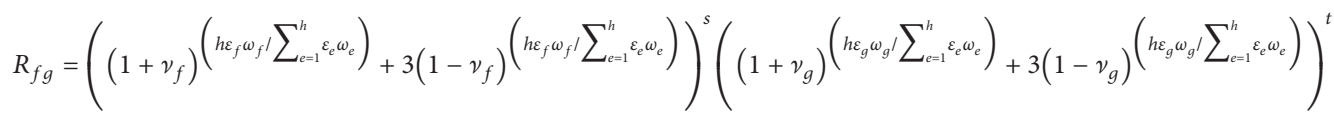

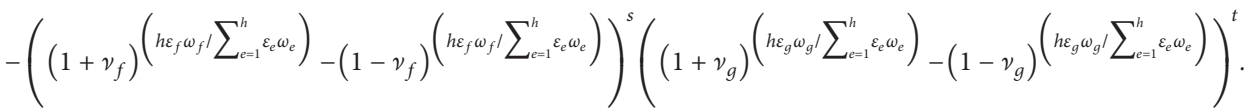

\section{MADM Model}

A novel MADM model using the proposed operators to manage PFNs is illustrated as follows. Suppose that there are $m$ alternatives represented by $L=\left\{L_{1}, L_{2}, \ldots, L_{m}\right\}$ and $h$ attributes represented by $A=\left\{A_{1}, A_{2}, \ldots, A_{h}\right\}$, and the importance of each attribute is denoted by $\omega_{f}(f=1,2, \ldots, h)$, satisfying $\sum_{f=1}^{h} \omega_{f}=1$ and $\omega_{f} \geq 0$. The assessment value of alternative $L_{i}$ relating to attribute $A_{f}$ takes the form of PFN: $\alpha_{i f}=\left\langle\mu\left(\alpha_{i f}\right), \eta\left(\alpha_{i f}\right), v\left(\alpha_{i f}\right)\right\rangle$ $(i=1,2, \ldots, m ; f=1,2, \ldots, h)$, where $0 \leq \mu\left(\alpha_{i f}\right), \eta\left(\alpha_{i f}\right)$, $\nu\left(\alpha_{i f}\right) \leq 1$ and $0 \leq \mu\left(\alpha_{i f}\right)+\eta\left(\alpha_{i f}\right)+\nu\left(\alpha_{i f}\right) \leq 1$. The initial matrix $D=\left[\alpha_{i f}\right]_{m \times h}(i=1,2, \ldots, m ; f=1,2, \ldots, h)$ is provided by experts.

The MADM model with PFN is shown in Figure 1 and the detailed steps are presented as follows.
Step 1. Obtain the normalized matrix $\widetilde{D}=\left[\beta_{i f}\right]_{m \times h}$. The assessment value $\alpha_{i f}$ with the cost attribute $A_{f}$ needs to be transformed into $\beta_{\text {if }}$ based on the following formula:

$\beta_{i f}= \begin{cases}\left\langle\mu\left(\alpha_{i f}\right), \eta\left(\alpha_{i f}\right), \nu\left(\alpha_{i f}\right)\right\rangle, & \text { for benefit attribute, } \\ \left\langle\nu\left(\alpha_{i f}\right), \eta\left(\alpha_{i f}\right), \mu\left(\alpha_{i f}\right)\right\rangle, & \text { for cost attribute. }\end{cases}$

Here, $i=1,2, \ldots, m ; f=1,2, \ldots, h$.

Step 2. Compute the supports $\sup \left(\beta_{i f}, \beta_{i g}\right)$.

The supports are calculated based on the following formula:

$$
\sup \left(\beta_{i f}, \beta_{i g}\right)=1-d\left(\beta_{i f}, \beta_{i g}\right)
$$




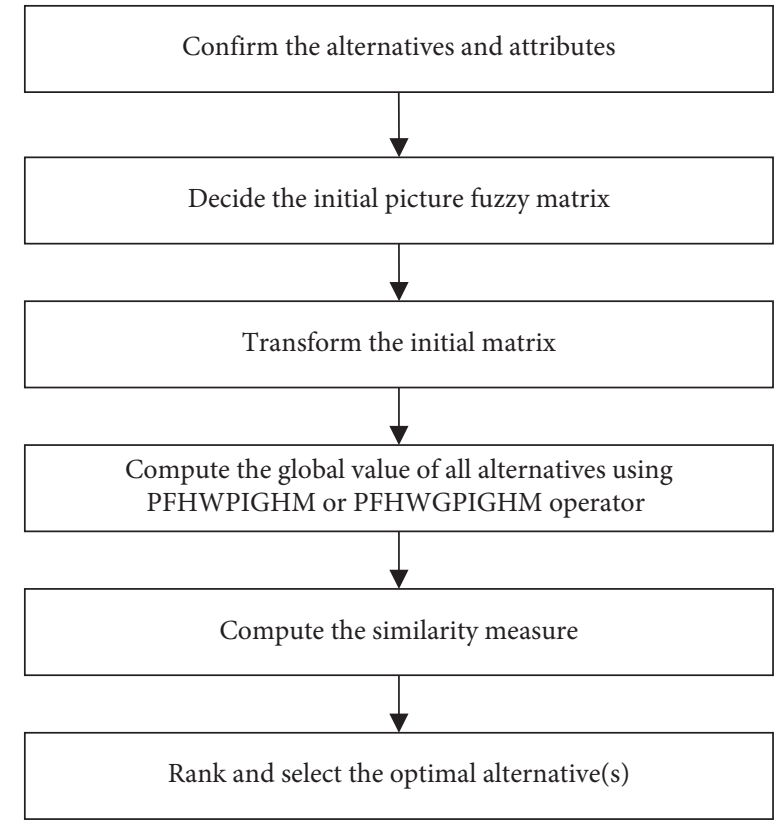

Figure 1: The proposed model.

Here, $i=1,2, \ldots, m ; f, g=1,2, \ldots, h$, and $d\left(\beta_{i f}, \beta_{i q}\right)$ is the normalized Hamming distance between PFNs $\beta_{i f}$ and $\beta_{i g}$ provided in Definition 3.

Step 3. Compute the power weights $\varepsilon_{i f}$.

$$
\varepsilon_{i f}=\frac{1+\varphi\left(\beta_{i f}\right)}{\sum_{e=1}^{h}\left(1+\varphi\left(\beta_{i e}\right)\right)},
$$

where $\varphi\left(\beta_{i f}\right)=\sum_{\substack{g=1 \\ g \neq f}}^{h}, \sup \left(\beta_{i f}, \beta_{i g}\right)$, and $i=1,2, \ldots$, $m ; f, g=1,2, \ldots, h$.

$$
, h \neq
$$

Step 4. Compute the weights $\zeta_{i f}$ of PFN $\beta_{i f}$.

$$
\zeta_{i f}=\frac{\varepsilon_{i f} \omega_{f}}{\sum_{e=1}^{h} \varepsilon_{i e} \omega_{e}}
$$

where $\zeta_{i f} \geq 0$ and $\sum_{f=1}^{h} \zeta_{i f}=1$, and $i=1,2, \ldots, m$; $f, g=1,2, \ldots, h$.
Step 5. Compute the global value $\beta_{i}$ of each alternative $L_{i}$.

The global value $\beta_{i}$ can be obtained utilizing the PFHWPIGHM operator or the PFHWGPIGHM operator as defined in Theorems 1 and 3.

$$
\beta_{i}=\operatorname{PFHWPIGHM}^{s, t}\left(\beta_{i 1}, \beta_{i 2}, \ldots, \beta_{i h}\right),
$$

or

$$
\beta_{i}=\operatorname{PFHWGPIGHM}^{s, t}\left(\beta_{i 1}, \beta_{i 2}, \ldots, \beta_{i h}\right) .
$$

Here $i=1,2, \ldots, m$.

Step 6. Compute the similarity of alternative $L_{i}$ and the ideal alternative $L^{*}$.

According to Definition 4, the similarity can be calculated based on the following formula:

$$
s\left(L, L^{*}\right)=\frac{\mu\left(\beta_{i}\right)}{\sqrt{\mu^{2}\left(\beta_{i}\right)+\eta^{2}\left(\beta_{i}\right)+\nu^{2}\left(\beta_{i}\right)}} .
$$

Step 7. Rank and select alternative(s).

Based on the similarity measure calculated above, all alternatives are ranked, and the best vendor is obtained.

\section{Example and Analysis}

An example based on the MADM approach proposed above is applied to verify its practicability, and corresponding analysis is conducted as well.

5.1. Example. Assume that a company wants to purchase an ERP system from five vendors represented by $L_{i}(i=1,2,3,4,5)$, and there are four attributes represented by $A_{f}(f=1,2,3,4)$ to evaluate different vendors, where $A_{1}$ denotes function, $A_{2}$ denotes fitness, $A_{3}$ denotes capability, and $A_{4}$ denotes reputation, whose weight vector is $\omega=(0.2,0.1,0.3,0.4)$. The assessment value of each vendor $L_{i}$ with attribute $A_{f}$ is denoted by $\mathrm{PFN} \alpha_{i f}$, and the matrix $D=\left[\alpha_{i f}\right]_{5 \times 4}$ below is provided:

$$
D=\left[\alpha_{i f}\right]_{5 \times 4}=\left[\begin{array}{cccc}
\langle 0.53,0.33,0.09\rangle & \langle 0.89,0.08,0.03\rangle & \langle 0.42,0.35,0.18\rangle & \langle 0.08,0.89,0.02\rangle \\
\langle 0.73,0.12,0.08\rangle & \langle 0.13,0.64,0.21,\rangle & \langle 0.03,0.82,0.13\rangle & \langle 0.73,0.15,0.08\rangle \\
\langle 0.91,0.03,0.02\rangle & \langle 0.07,0.09,0.05\rangle & \langle 0.04,0.85,0.10\rangle & \langle 0.68,0.26,0.06\rangle \\
\langle 0.85,0.09,0.05\rangle & \langle 0.74,0.16,0.10\rangle & \langle 0.02,0.89,0.05\rangle & \langle 0.08,0.84,0.06\rangle \\
\langle 0.90,0.05,0.02\rangle & \langle 0.68,0.08,0.21\rangle & \langle 0.05,0.87,0.06\rangle & \langle 0.13,0.75,0.09\rangle
\end{array}\right]
$$

To choose the best vendor, the detailed steps are shown as follows:

Step 1. Obtain the normalized matrix $\widetilde{D}=\left[\beta_{i f}\right]_{5 \times 4}$.
Since all attributes are benefit type, the normalized matrix $\widetilde{D}=\left[\beta_{i f}\right]_{5 \times 4}$ is equal to the original matrix $D=\left[\alpha_{i f}\right]_{5 \times 4}$.

Step 2. Compute the supports $\sup \left(\beta_{i f}, \beta_{i g}\right)$. 
Based on the normalized Hamming distance between PFNs $\beta_{i f}$ and $\beta_{i g}$ in Definition 3, we can get

$$
\begin{aligned}
& d\left(\beta_{11}, \beta_{12}\right)=0.335, \\
& d\left(\beta_{11}, \beta_{13}\right)=0.110, \\
& d\left(\beta_{11}, \beta_{14}\right)=0.540, \\
& d\left(\beta_{12}, \beta_{13}\right)=0.445, \\
& d\left(\beta_{12}, \beta_{14}\right)=0.815, \\
& d\left(\beta_{13}, \beta_{14}\right)=0.520, \\
& d\left(\beta_{21}, \beta_{22}\right)=0.625, \\
& d\left(\beta_{21}, \beta_{23}\right)=0.725, \\
& d\left(\beta_{21}, \beta_{24}\right)=0.015, \\
& d\left(\beta_{22}, \beta_{23}\right)=0.180, \\
& d\left(\beta_{22}, \beta_{24}\right)=0.610, \\
& d\left(\beta_{23}, \beta_{24}\right)=0.710, \\
& d\left(\beta_{31}, \beta_{32}\right)=0.465, \\
& d\left(\beta_{31}, \beta_{33}\right)=0.885, \\
& d\left(\beta_{31}, \beta_{34}\right)=0.250, \\
& d\left(\beta_{32}, \beta_{33}\right)=0.420, \\
& d\left(\beta_{32}, \beta_{34}\right)=0.395, \\
& d\left(\beta_{33}, \beta_{34}\right)=0.635, \\
& d\left(\beta_{41}, \beta_{42}\right)=0.115, \\
& d\left(\beta_{41}, \beta_{43}\right)=0.815, \\
& d\left(\beta_{41}, \beta_{44}\right)=0.765, \\
& d\left(\beta_{42}, \beta_{43}\right)=0.750, \\
& d\left(\beta_{42}, \beta_{44}\right)=0.690, \\
& d\left(\beta_{43}, \beta_{44}\right)=0.060, \\
& d\left(\beta_{51}, \beta_{52}\right)=0.220, \\
& d\left(\beta_{51}, \beta_{53}\right)=0.855, \\
& d\left(\beta_{51}, \beta_{54}\right)=0.770, \\
& d\left(\beta_{52}, \beta_{53}\right)=0.785, \\
& d\left(\beta_{52}, \beta_{54}\right)=0.670, \\
& d\left(\beta_{53}, \beta_{54}\right)=0.115 .
\end{aligned}
$$

Then,

$$
\begin{aligned}
& \sup \left(\beta_{11}, \beta_{12}\right)=\sup \left(\beta_{12}, \beta_{11}\right)=0.665, \\
& \sup \left(\beta_{11}, \beta_{13}\right)=\sup \left(\beta_{13}, \beta_{11}\right)=0.890, \\
& \sup \left(\beta_{11}, \beta_{14}\right)=\sup \left(\beta_{14}, \beta_{11}\right)=0.460, \\
& \sup \left(\beta_{12}, \beta_{13}\right)=\sup \left(\beta_{13}, \beta_{12}\right)=0.555, \\
& \sup \left(\beta_{12}, \beta_{14}\right)=\sup \left(\beta_{14}, \beta_{12}\right)=0.185, \\
& \sup \left(\beta_{13}, \beta_{14}\right)=\sup \left(\beta_{14}, \beta_{13}\right)=0.480, \\
& \sup \left(\beta_{21}, \beta_{22}\right)=\sup \left(\beta_{22}, \beta_{21}\right)=0.375, \\
& \sup \left(\beta_{21}, \beta_{23}\right)=\sup \left(\beta_{23}, \beta_{21}\right)=0.275, \\
& \sup \left(\beta_{21}, \beta_{24}\right)=\sup \left(\beta_{24}, \beta_{21}\right)=0.985, \\
& \sup \left(\beta_{22}, \beta_{23}\right)=\sup \left(\beta_{23}, \beta_{22}\right)=0.820, \\
& \sup \left(\beta_{22}, \beta_{24}\right)=\sup \left(\beta_{24}, \beta_{22}\right)=0.390, \\
& \sup \left(\beta_{23}, \beta_{24}\right)=\sup \left(\beta_{24}, \beta_{23}\right)=0.290, \\
& \sup \left(\beta_{31}, \beta_{32}\right)=\sup \left(\beta_{32}, \beta_{31}\right)=0.535, \\
& \sup \left(\beta_{31}, \beta_{33}\right)=\sup \left(\beta_{33}, \beta_{31}\right)=0.115, \\
& \sup \left(\beta_{31}, \beta_{34}\right)=\sup \left(\beta_{34}, \beta_{31}\right)=0.750, \\
& \sup \left(\beta_{32}, \beta_{33}\right)=\sup \left(\beta_{33}, \beta_{32}\right)=0.580, \\
& \sup \left(\beta_{32}, \beta_{34}\right)=\sup \left(\beta_{34}, \beta_{32}\right)=0.605, \\
& \sup \left(\beta_{33}, \beta_{34}\right)=\sup \left(\beta_{34}, \beta_{33}\right)=0.365, \\
& \sup \left(\beta_{41}, \beta_{42}\right)=\sup \left(\beta_{42}, \beta_{41}\right)=0.885, \\
& \sup \left(\beta_{41}, \beta_{43}\right)=\sup \left(\beta_{43}, \beta_{41}\right)=0.185, \\
& \sup \left(\beta_{41}, \beta_{44}\right)=\sup \left(\beta_{44}, \beta_{41}\right)=0.235, \\
& \sup \left(\beta_{42}, \beta_{43}\right)=\sup \left(\beta_{43}, \beta_{42}\right)=0.250, \\
& \sup \left(\beta_{42}, \beta_{44}\right)=\sup \left(\beta_{44}, \beta_{42}\right)=0.310, \\
& \sup \left(\beta_{43}, \beta_{44}\right)=\sup \left(\beta_{44}, \beta_{43}\right)=0.940, \\
& \sup \left(\beta_{51}, \beta_{52}\right)=\sup \left(\beta_{52}, \beta_{51}\right)=0.780, \\
& \sup \left(\beta_{51}, \beta_{53}\right)=\sup \left(\beta_{53}, \beta_{51}\right)=0.145, \\
& \sup \left(\beta_{51}, \beta_{54}\right)=\sup \left(\beta_{54}, \beta_{51}\right)=0.230, \\
& \sup \left(\beta_{52}, \beta_{53}\right)=\sup \left(\beta_{53}, \beta_{52}\right)=0.215, \\
& \sup \left(\beta_{52}, \beta_{54}\right)=\sup \left(\beta_{54}, \beta_{52}\right)=0.330, \\
& \sup \left(\beta_{53}, \beta_{54}\right)=\sup \left(\beta_{54}, \beta_{53}\right)=0.885 .
\end{aligned}
$$


Step 3. Compute the power weights $\varepsilon_{i f}$.

As an example, $\varphi\left(\beta_{11}\right)$ can be computed as follows based on the formula $\varphi\left(\beta_{i f}\right)=\sum_{\substack{g=1 \\ g \neq f}}^{h}, \sup \left(\beta_{i f}, \beta_{i g}\right)$ :

$$
\begin{aligned}
\varphi\left(\beta_{11}\right) & =\sup \left(\beta_{11}, \beta_{12}\right)+\sup \left(\beta_{11}, \beta_{13}\right)+\sup \left(\beta_{11}, \beta_{14}\right) \\
& =0.665+0.89+0.46 \\
& =2.015 .
\end{aligned}
$$

Then, we can get

$$
\left[\varphi\left(\beta_{i f}\right)\right]_{5 \times 4}=\left[\begin{array}{cccc}
2.015 & 1.405 & 1.925 & 1.125 \\
1.635 & 1.585 & 1.385 & 1.665 \\
1.4 & 1.72 & 1.06 & 1.72 \\
1.305 & 1.445 & 1.375 & 1.485 \\
1.155 & 1.325 & 1.245 & 1.445
\end{array}\right] .
$$

Then, based on formula (33), we can get

$$
\left[\varepsilon_{i f}\right]_{5 \times 4}=\left[\begin{array}{llll}
0.2880 & 0.2297 & 0.2794 & 0.2030 \\
0.2566 & 0.2517 & 0.2322 & 0.2595 \\
0.2424 & 0.2747 & 0.2081 & 0.2747 \\
0.2399 & 0.2544 & 0.2471 & 0.2586 \\
0.2350 & 0.2535 & 0.2448 & 0.2666
\end{array}\right] \text {. }
$$

Step 4. Compute the weights $\zeta_{\text {if }}$ of $\mathrm{PFN} \beta_{i f}$. Based on formula (34), we can get

$$
\left[\zeta_{i f}\right]_{5 \times 4}=\left[\begin{array}{llll}
0.2345 & 0.0935 & 0.3413 & 0.3306 \\
0.2053 & 0.1007 & 0.2787 & 0.4153 \\
0.1953 & 0.1107 & 0.2514 & 0.4426 \\
0.1911 & 0.1014 & 0.2954 & 0.4121 \\
0.1862 & 0.1004 & 0.2909 & 0.4225
\end{array}\right] .
$$

Step 5. Compute the global value $\beta_{i}$ of each alternative $L_{i}$. Let $\gamma=2, s=t=1$. The global value $\beta_{i}$ can be obtained utilizing the PFHWPIGHM operator as defined in Theorem 1 .

$$
\begin{aligned}
& \beta_{1}=\langle 0.3779,0.4754,0.0420\rangle, \\
& \beta_{2}=\langle 0.6544,0.2185,0.0637\rangle, \\
& \beta_{3}=\langle 0.6804,0.2195,0.0374\rangle, \\
& \beta_{4}=\langle 0.2992,0.6013,0.0335\rangle, \\
& \beta_{5}=\langle 0.3504,0.5146,0.0388\rangle .
\end{aligned}
$$

Similarly, the global value $\beta_{i}$ can be obtained utilizing the PFHWGPIGHM operator as defined in Theorem 3.

$$
\begin{aligned}
& \beta_{1}=\langle 0.3214,0.5766,0.1029\rangle, \\
& \beta_{2}=\langle 0.3678,0.4453,0.1110\rangle, \\
& \beta_{3}=\langle 0.3665,0.4298,0.0665\rangle, \\
& \beta_{4}=\langle 0.1872,0.7498,0.0649\rangle, \\
& \beta_{5}=\langle 0.2426,0.6444,0.0870\rangle .
\end{aligned}
$$

Step 6. Compute the similarity of alternative $L_{i}$ and the ideal alternative $L^{*}$.

The similarity can be calculated based on formula (37). For the PFHWPIGHM operator,

$$
\begin{gathered}
s\left(L_{1}, L^{*}\right)=0.6208, \\
s\left(L_{2}, L^{*}\right)=0.9445, \\
s\left(L_{3}, L^{*}\right)=0.9504, \\
s\left(L_{4}, L^{*}\right)=0.4449, \\
s\left(L_{5}, L^{*}\right)=0.5617 .
\end{gathered}
$$

For the PFHWGPIGHM operator,

$$
\begin{gathered}
s\left(L_{1}, L^{*}\right)=0.4811, \\
s\left(L_{2}, L^{*}\right)=0.6254, \\
s\left(L_{3}, L^{*}\right)=0.6444, \\
s\left(L_{4}, L^{*}\right)=0.2414, \\
s\left(L_{5}, L^{*}\right)=0.3496 .
\end{gathered}
$$

Step 7. Rank and select alternative(s).

The final ranking using the PFHWPIGHM operator or the PFHWGPIGHM operator is always $L_{3}>L_{2}>L_{1}>L_{5}>L_{4}$, and the best vendor is $L_{3}$, while $L_{4}$ is the worst one.

5.2. Sensitivity Analysis. In this part, we will discuss the impact of parameters $\gamma, \mathrm{s}$, and $t$ to the final ranking result.

When parameters $s=t=1, \gamma$ is assigned to different values, and the rankings utilizing the PFHWPIGHM operator and the PFHWGPIGHM operator are presented in Tables 1 and 2, respectively.

From Tables 1 and 2, it can be clearly observed that the worst vendor is always $L_{4}$, while the best vendor is always $L_{3}$ utilizing the PFHWPIGHM operator, and the best vendor is $L_{3}$ or $L_{2}$ utilizing the PFHWGPIGHM operator.

When parameter $\gamma=1, s$ and $t$ are assigned to different values, and the rankings utilizing the PFHWPIGHM operator and the PFHWGPIGHM operator are presented in Tables 3 and 4, respectively.

From Tables 3 and 4, it can be clearly observed that the worst vendor is always $L_{4}$ utilizing the PFHWGPIGHM operator, while the best vendor is $L_{3}$ or $L_{2}$ utilizing the PFHWPIGHM operator with different parameters $s$ and $t$. 
TABLE 1: Rankings based on the PFHWPIGHM operator with different parameter $\gamma$.

\begin{tabular}{lc}
\hline$\gamma$ & Rankings \\
\hline 1 & $L_{3}>L_{2}>L_{1}>L_{5}>L_{4}$ \\
2 & $L_{3}>L_{2}>L_{1}>L_{5}>L_{4}$ \\
3 & $L_{3}>L_{2}>L_{1}>L_{5}>L_{4}$ \\
5 & $L_{3}>L_{2}>L_{1}>L_{5}>L_{4}$ \\
10 & $L_{3}>L_{2}>L_{1}>L_{5}>L_{4}$ \\
15 & $L_{3}>L_{2}>L_{1}>L_{5}>L_{4}$ \\
20 & $L_{3}>L_{2}>L_{1}>L_{5}>L_{4}$ \\
50 & $L_{3}>L_{2}>L_{1}>L_{5}>L_{4}$ \\
\hline
\end{tabular}

TABle 2: Rankings based on the PFHWGPIGHM operator with different parameter $\gamma$.

\begin{tabular}{lc}
\hline$\gamma$ & Rankings \\
\hline 1 & $L_{3}>L_{2}>L_{1}>L_{5}>L_{4}$ \\
2 & $L_{3}>L_{2}>L_{1}>L_{5}>L_{4}$ \\
3 & $L_{3}>L_{2}>L_{1}>L_{5}>L_{4}$ \\
5 & $L_{3}>L_{2}>L_{1}>L_{5}>L_{4}$ \\
10 & $L_{3}>L_{2}>L_{1}>L_{5}>L_{4}$ \\
15 & $L_{2}>L_{3}>L_{1}>L_{5}>L_{4}$ \\
20 & $L_{2}>L_{3}>L_{1}>L_{5}>L_{4}$ \\
50 & $L_{2}>L_{3}>L_{1}>L_{5}>L_{4}$ \\
\hline
\end{tabular}

TABLE 3: Rankings based on the PFHWPIGHM operator with $\gamma=1$.

\begin{tabular}{lc}
\hline$s, t$ & \multicolumn{1}{c}{ Rankings } \\
\hline$s=t=0.5$ & $L_{3}>L_{2}>L_{1}>L_{5}>L_{4}$ \\
$s=0.5, t=0$ & $L_{3}>L_{2}>L_{5}>L_{1}>L_{4}$ \\
$s=1, t=0$ & $L_{3}>L_{2}>L_{5}>L_{1}>L_{4}$ \\
$s=0, t=0.5$ & $L_{2}>L_{3}>L_{1}>L_{5}>L_{4}$ \\
$s=0, t=1$ & $L_{2}>L_{3}>L_{1}>L_{5}>L_{4}$ \\
$s=t=1$ & $L_{3}>L_{2}>L_{1}>L_{5}>L_{4}$ \\
$s=2, t=1$ & $L_{3}>L_{2}>L_{5}>L_{1}>L_{4}$ \\
$s=5, t=1$ & $L_{3}>L_{2}>L_{5}>L_{4}>L_{1}$ \\
$s=1, t=2$ & $L_{2}>L_{3}>L_{1}>L_{5}>L_{4}$ \\
$s=1, t=5$ & $L_{2}>L_{3}>L_{5}>L_{1}>L_{4}$ \\
$s=t=2$ & $L_{3}>L_{2}>L_{5}>L_{1}>L_{4}$ \\
$s=t=5$ & $L_{2}>L_{3}>L_{5}>L_{4}>L_{1}$ \\
$s=t=10$ & $L_{2}>L_{3}>L_{5}>L_{4}>L_{1}$ \\
\hline
\end{tabular}

TABle 4: Rankings based on the PFHWGPIGHM operator with $\gamma=1$

\begin{tabular}{lc}
\hline$s, t$ & \multicolumn{1}{c}{ Rankings } \\
\hline$s=t=0.5$ & $L_{3}>L_{2}>L_{1}>L_{5}>L_{4}$ \\
$s=0.5, t=0$ & $L_{3}>L_{1}>L_{5}>L_{2}>L_{4}$ \\
$s=1, t=0$ & $L_{1}>L_{3}>L_{2}>L_{5}>L_{4}$ \\
$s=0, t=0.5$ & $L_{3}>L_{2}>L_{1}>L_{5}>L_{4}$ \\
$s=0, t=1$ & $L_{3}>L_{2}>L_{1}>L_{5}>L_{4}$ \\
$s=t=1$ & $L_{3}>L_{2}>L_{1}>L_{5}>L_{4}$ \\
$s=2, t=1$ & $L_{2}>L_{3}>L_{1}>L_{5}>L_{4}$ \\
$s=5, t=1$ & $L_{3}>L_{2}>L_{1}>L_{5}>L_{4}$ \\
$s=1, t=2$ & $L_{3}>L_{2}>L_{1}>L_{5}>L_{4}$ \\
$s=1, t=5$ & $L_{3}>L_{2}>L_{1}>L_{5}>L_{4}$ \\
$s=t=2$ & $L_{3}>L_{2}>L_{1}>L_{5}>L_{4}$ \\
$s=t=5$ & $L_{3}>L_{2}>L_{1}>L_{5}>L_{4}$ \\
$s=t=10$ & $L_{3}>L_{2}>L_{1}>L_{5}>L_{4}$ \\
\hline
\end{tabular}

TABLE 5: Rankings based on the PFHWPIGHM operator with $\gamma=2$.

\begin{tabular}{lc}
\hline$s, t$ & \multicolumn{1}{c}{ Rankings } \\
\hline$s=t=0.5$ & $L_{3}>L_{2}>L_{1}>L_{5}>L_{4}$ \\
$s=0.5, t=0$ & $L_{3}>L_{2}>L_{1}>L_{5}>L_{4}$ \\
$s=1, t=0$ & $L_{3}>L_{2}>L_{1}>L_{5}>L_{4}$ \\
$s=0, t=0.5$ & $L_{2}>L_{3}>L_{1}>L_{5}>L_{4}$ \\
$s=0, t=1$ & $L_{2}>L_{3}>L_{1}>L_{5}>L_{4}$ \\
$s=t=1$ & $L_{3}>L_{2}>L_{1}>L_{5}>L_{4}$ \\
$s=2, t=1$ & $L_{3}>L_{2}>L_{5}>L_{1}>L_{4}$ \\
$s=5, t=1$ & $L_{3}>L_{2}>L_{5}>L_{4}>L_{1}$ \\
$s=1, t=2$ & $L_{2}>L_{3}>L_{1}>L_{5}>L_{4}$ \\
$s=1, t=5$ & $L_{2}>L_{3}>L_{5}>L_{1}>L_{4}$ \\
$s=t=2$ & $L_{3}>L_{2}>L_{5}>L_{1}>L_{4}$ \\
$s=t=5$ & $L_{2}>L_{3}>L_{5}>L_{4}>L_{1}$ \\
$s=t=10$ & $L_{2}>L_{3}>L_{5}>L_{4}>L_{1}$ \\
\hline
\end{tabular}

TABLE 6: Rankings based on the PFHWGPIGHM operator with $\gamma=2$.

\begin{tabular}{lc}
\hline$s, t$ & \multicolumn{1}{c}{ Rankings } \\
\hline$s=t=0.5$ & $L_{3}>L_{2}>L_{1}>L_{5}>L_{4}$ \\
$s=0.5, t=0$ & $L_{3}>L_{1}>L_{5}>L_{2}>L_{4}$ \\
$s=1, t=0$ & $L_{3}>L_{1}>L_{2}>L_{5}>L_{4}$ \\
$s=0, t=0.5$ & $L_{3}>L_{2}>L_{1}>L_{5}>L_{4}$ \\
$s=0, t=1$ & $L_{3}>L_{2}>L_{1}>L_{5}>L_{4}$ \\
$s=t=1$ & $L_{3}>L_{2}>L_{1}>L_{5}>L_{4}$ \\
$s=2, t=1$ & $L_{2}>L_{3}>L_{1}>L_{5}>L_{4}$ \\
$s=5, t=1$ & $L_{3}>L_{2}>L_{1}>L_{5}>L_{4}$ \\
$s=1, t=2$ & $L_{3}>L_{2}>L_{1}>L_{5}>L_{4}$ \\
$s=1, t=5$ & $L_{3}>L_{2}>L_{1}>L_{5}>L_{4}$ \\
$s=t=2$ & $L_{3}>L_{2}>L_{1}>L_{5}>L_{4}$ \\
$s=t=5$ & $L_{3}>L_{2}>L_{1}>L_{5}>L_{4}$ \\
$s=t=10$ & $L_{3}>L_{2}>L_{1}>L_{5}>L_{4}$ \\
\hline
\end{tabular}

TABLE 7: Rankings of different operators.

\begin{tabular}{lc}
\hline Operator & Ranking \\
\hline PFHWA [45], $\gamma=2$ & $L_{3}>L_{2}>L_{1}>L_{5}>L_{4}$ \\
PFHWG [45], $\gamma=2$ & $L_{3}>L_{1}>L_{2}>L_{5}>L_{4}$ \\
PFNWBM [41], $s=t=1$ & $L_{3}>L_{2}>L_{1}>L_{5}>L_{4}$ \\
PFHWPIGHM, $\gamma=2, s=t=1$ & $L_{3}>L_{2}>L_{1}>L_{5}>L_{4}$ \\
PFHWGPIGHM, $\gamma=2, s=t=1$ & $L_{3}>L_{2}>L_{1}>L_{5}>L_{4}$ \\
\hline
\end{tabular}

When parameter $\gamma=2, s$ and $t$ are assigned to different values, and the rankings utilizing the PFHWPIGHM operator and the PFHWGPIGHM operator are presented in Tables 5 and 6 , respectively.

From Tables 5 and 6, it can be clearly observed that the best vendor is $L_{3}$ or $L_{2}$, while the worst vendor is always $L_{4}$ utilizing the PFHWGPIGHM operator, and the worst vendor is $L_{4}$ or $L_{1}$ utilizing the PFHWPIGHM operator with different parameters $s$ and $t$.

From the sensitivity analysis above, we know that different parameters in the PFHWPIGHM operator and the PFHWGPIGHM operator may cause different ranking orders; thus, parameters $\gamma, s$, and $t$ play an essential part in the 
TABle 8: Comparison of characteristics between different methods.

\begin{tabular}{lccc}
\hline Characteristic & Wei [45] & Ates and Akay [41] & Proposed method \\
\hline Consider correlations of attributes & No & Yes & Yes \\
Reduce the influence of biased data & No & No & Yes \\
Operational rules & Hamacher & Algebraic & Hamacher \\
Number of parameters & $\gamma$ & $s, t$ & $\gamma, s, t$ \\
\hline
\end{tabular}

MADM problem. When $\gamma=1$ or $\gamma=2$, picture fuzzy Hamacher operations can be degenerated to picture fuzzy Algebraic or Einstein operations, respectively. Therefore, the picture fuzzy Hamacher operators proposed in this paper are more general. Furthermore, parameter $s$ or $t$ can illustrate the risk preference of DMs who can decide the value of parameter $s$ or $t$ in terms of actual situation. Generally, the risk seeker will assign big value to parameter $s$ or $t$. Therefore, the PFHWPIGHM operator and the PFHWGPIGHM operator are more flexible.

5.3. Comparison Analysis. To further demonstrate the superiorities of the proposed MADM method using the PFHWPIGHM operator and the PFHWGPIGHM operator, we compare the method proposed in this paper with the existing method proposed by Wei [45], based on the PFHWA operator and the PFHWG operator, and the method proposed by Ates and Akay [41], based on the PFNWBM operator. The rankings of different AOs are listed in Table 7.

From Table 7, it is clearly observed that the ranking orders of different AOs are the same except the PFHWG operator. Besides, the best vendor is always $L_{3}$, and the worst one is always $L_{4}$ for all AOs presented above.

Furthermore, a comparison between our method and these methods mentioned above as regards some characteristics is presented in Table 8 .

The method of Wei [45] does not consider the correlations of attributes and the influence of biased data, and only one parameter is considered. The method of Ates and Akay [41] considers the correlations of attributes but cannot reduce the influence of biased data, and it is based on Algebraic operations, which is only a special case of Hamacher operations, and only two parameters are considered. In contrast, our method not only considers the correlations of attributes but also reduces the influence of biased data. Moreover, it is based on Hamacher operational rules and three parameters $\gamma, s$, and $t$. These characteristics make the proposed method more general and flexible.

\section{Conclusion and Future Directions}

On the basis of the related studies of AOs, the PIGHM operators, which combine the PA operator and the IGHM operator, are firstly explored in this paper. Considering the superiorities of PFS and Hamacher operations, two novel AOs, the PFHWPIGHM operator and the PFHWGPIGHM operator, are proposed, and some particular cases are also discussed. Further, based on these proposed AOs, a MADM model is constructed under PFS environment, and the detailed steps are also investigated. Eventually, an example on ERP vendor selection is conducted to demonstrate the practicality and credibility of the proposed model. Besides, we discuss the impact of different parameters $\gamma, s$, and $t$ on the final ranking, and the comparable analysis from different aspects is also concluded.

In the recent years, to better convey the vagueness and uncertainty of cognitive information, some different fuzzy sets and their applications have been proposed and developed, such as Pythagorean fuzzy set [47], intuitionistic fuzzy set [48], Pythagorean 2-tuple linguistic set [49], probabilistic linguistic set [50], picture fuzzy labelling of graphs [51], picture fuzzy soft set [52], bipolar picture fuzzy set [53], intuitionistic multiplicative set [54], and $t$-spherical fuzzy set [55]. In future research, it is meaningful to explore the application of the proposed AOs and MADM model to these fuzzy sets and apply them to different fields.

\section{Data Availability}

The data used to support the findings of this study are included within the article.

\section{Conflicts of Interest}

The authors declare that there are no conflicts of interest.

\section{Acknowledgments}

This research was funded by the Social Science Foundation of Hubei Province under Grant no. 20ZD065 and Philosophy and Social Science Research Project of Hubei Province under Grant no. 20y111.

\section{References}

[1] K. T. Atanassov, "Intuitionistic fuzzy sets," Fuzzy Sets and Systems, vol. 20, no. 1, pp. 87-96, 1986.

[2] L. A. Zadeh, "Fuzzy sets," Information and Control, vol. 8, no. 2, pp. 338-356, 1965

[3] B. C. Cuong, "Picture fuzzy sets-a new concept for computational intelligence problems," in Proceedings of the 2013 Third World Congress on Information and Communication Technologies (WICT 2013), pp. 1-3, Hanoi, Vietnam, December 2013.

[4] H. Garg, "Some picture fuzzy aggregation operators and their applications to multicriteria decision-making," Arabian Journal for Science and Engineering, vol. 42, no. 12, pp. 5275-5290, 2017.

[5] L. H. Son, "Generalized picture distance measure and applications to picture fuzzy clustering," Applied Soft Computing, vol. 46, pp. 284-295, 2016. 
[6] M. Luo and Y. Zhang, "A new similarity measure between picture fuzzy sets and its application," Engineering Applications of Artificial Intelligence, vol. 96, Article ID 103956, 2020.

[7] P. Liu, M. Akram, and A. Bashir, "Extensions of power aggregation operators for decision making based on complex picture fuzzy knowledge," Journal of Intelligent \& Fuzzy Systems, vol. 40, no. 1, pp. 1107-1128, 2021.

[8] A. H. Ganie and S. Singh, "An innovative picture fuzzy distance measure and novel multi-attribute decision-making method," Complex \& Intelligent Systems, vol. 7, no. 2, pp. 781-805, 2021.

[9] L. Yang and B. Li, "Multiple-valued picture fuzzy linguistic set based on generalized heronian mean operators and their applications in multiple attribute decision making," IEEE Access, vol. 8, pp. 86272-86295, 2020.

[10] R. R. Yager, “The power average operator," IEEE Transactions on Systems, Man, and Cybernetics - Part A: Systems and Humans, vol. 31, no. 6, pp. 724-731, 2001.

[11] D. Rani and H. Garg, "Complex intuitionistic fuzzy power aggregation operators and their applications in multicriteria decisionmaking," Expert Systems, vol. 35, no. 6, Article ID e12325, 2018.

[12] W. Jiang, B. Wei, X. Liu, X. Li, and H. Zheng, "Intuitionistic fuzzy power aggregation operator based on entropy and its application in decision making," International Journal of Intelligent Systems, vol. 33, no. 1, pp. 49-67, 2018.

[13] Y. Ju, C. Luo, J. Ma, and A. Wang, "A novel multiple-attribute group decision-making method based on $\mathrm{q}$-rung orthopair fuzzy generalized power weighted aggregation operators," International Journal of Intelligent Systems, vol. 34, no. 9, pp. 2077-2103, 2019.

[14] L. Yang and B. Li, "Multi-valued neurosophic linguistic power operators and their applications," Engineering Letters, vol. 26, pp. 518-525, 2018.

[15] H. Garg and K. Kumar, "Multiattribute decision making based on power operators for linguistic intuitionistic fuzzy set using set pair analysis," Expert Systems, vol. 36, no. 4, p. e12428, 2019.

[16] C. Liu and Y. Luo, "Power aggregation operators of simplified neutrosophic sets and their use in multi-attribute group decision making," IEEE/CAA Journal of Automatica Sinica, vol. 6 , no. 2, pp. 575-583, 2019.

[17] H. Garg and Nancy, "Linguistic single-valued neutrosophic power aggregation operators and their applications to group decision-making problems," IEEE/CAA Journal of Automatica Sinica, vol. 7, no. 2, pp. 546-558, 2020.

[18] G. Beliakov, A. Pradera, and T. Calvo, "Aggregation functions: a guide for practitioners," in Studies in Fuzziness and Soft ComputingSpringer-Verlag, Berlin, Germany, 2007.

[19] S. Sykora, Mathematical Means and Averages: Generalized Heronian Means, Vol. 3, Stan's Library, Castano Primo, Italy, 2009.

[20] D. J. Yu and Y. Y. Wu, "Interval-valued intuitionistic fuzzy heronian mean operators and their application in multicriteria decision making," African Journal of Business Management, vol. 6, no. 11, pp. 4158-4168, 2012.

[21] $\mathrm{D} . \mathrm{Yu}$, "Intuitionistic fuzzy geometric heronian mean aggregation operators," Applied Soft Computing, vol. 13, no. 2, pp. 1235-1246, 2013.

[22] Y. Chu and P. Liu, "Some two-dimensional uncertain linguistic heronian mean operators and their application in multiple-attribute decision making," Neural Computing and Applications, vol. 26, no. 6, pp. 1461-1480, 2015.

[23] Y. Li, P. Liu, and Y. Chen, "Some single valued neutrosophic number heronian mean operators and their application in multiple attribute group decision making," Informatica, vol. 27, no. 1, pp. 85-110, 2016.
[24] P. Liu and L. Zhang, "Multiple criteria decision making method based on neutrosophic hesitant fuzzy Heronian mean aggregation operators," Journal of Intelligent \& Fuzzy Systems, vol. 32, no. 1, pp. 303-319, 2017.

[25] P. Liu and F. Teng, "Multiple attribute group decision making methods based on some normal neutrosophic number Heronian Mean operators," Journal of Intelligent \& Fuzzy Systems, vol. 32, no. 3, pp. 2375-2391, 2017.

[26] P. Liu and L. Shi, "Some neutrosophic uncertain linguistic number Heronian mean operators and their application to multi-attribute group decision making," Neural Computing and Applications, vol. 28, no. 5, pp. 1079-1093, May. 2017.

[27] C. Fan, J. Ye, S. Feng, E. Fan, and K. Hu, "Multi-Criteria decision-making method using heronian mean operators under a bipolar neutrosophic environment," Mathematics, vol. 7, no. 1, p. 97, 2019.

[28] P. Liu, "Multiple attribute group decision making method based on interval-valued intuitionistic fuzzy power Heronian aggregation operators," Computers \& Industrial Engineering, vol. 108, pp. 199-212, 2017.

[29] M. Shi, F. Yang, and Y. Xiao, "Intuitionistic fuzzy power geometric Heronian mean operators and their application to multiple attribute decision making," Journal of Intelligent \& Fuzzy Systems, vol. 37, no. 2, pp. 2651-2669, 2019.

[30] S. Zhao, D. Wang, C. Liang, Y. Leng, and J. Xu, "Some singlevalued neutrosophic power heronian aggregation operators and their application to multiple-attribute group decisionmaking," Symmetry, vol. 11, no. 5, p. 653, 2019.

[31] S. Jiang, W. He, F. Qin, and Q. Cheng, "Multiple attribute group decision-making based on power heronian aggregation operators under interval-valued dual hesitant fuzzy environment," Mathematical Problems in Engineering, vol. 2020, Article ID 2080413, 19 pages, 2020.

[32] D. Ju, Y. Ju, and A. Wang, "Multi-attribute group decision making based on power generalized Heronian mean operator under hesitant fuzzy linguistic environment," Soft Computing, vol. 23, no. 11, pp. 3823-3842, 2019.

[33] J. Wang, P. Wang, G. Wei, C. Wei, and J. Wu, "Some power Heronian mean operators in multiple attribute decisionmaking based on q-rung orthopair hesitant fuzzy environment," Journal of Experimental \& Theoretical Artificial Intelligence, vol. 32, no. 6, pp. 909-937, 2019.

[34] D. Dordevic, G. Stojic, Z. Stevic, D. Pamucar, A. Vulevic, and V. Misic, "A new model for defining the criteria of service quality in rail transport: the full consistency method based on a rough power heronian aggregator," Symmetry-Basel, vol. 11, p. 992, 2019.

[35] Y. Zhong, H. Gao, X. Guo, Y. Qin, M. Huang, and X. Luo, "Dombi power partitioned Heronian mean operators of q-rung orthopair fuzzy numbers for multiple attribute group decision making," PLoS One, vol. 14, no. 10, Article ID e0222007, 2019.

[36] K. Bai, X. Zhu, J. Wang, and R. Zhang, "Power partitioned Heronian mean operators for $\mathrm{q}$-rung orthopair uncertain linguistic sets with their application to multiattribute group decision making," International Journal of Intelligent Systems, vol. 35, no. 1, pp. 3-37, 2019.

[37] R. Wang, J. Wang, H. Gao, and G. Wei, "Methods for MADM with picture fuzzy muirhead mean operators and their application for evaluating the financial investment risk," Symmetry, vol. 11, no. 1, p. 6, 2019.

[38] C. Jana, T. Senapati, M. Pal, and R. R. Yager, "Picture fuzzy Dombi aggregation operators: application to MADM process," Applied Soft Computing, vol. 74, pp. 99-109, 2019. 
[39] P. Liu and X. Zhang, "A novel picture fuzzy linguistic aggregation operator and its application to group decision-making," Cognitive Computation, vol. 10, no. 2, pp. 242-259, 2018.

[40] H. Zhang, R. Zhang, H. Huang, and J. Wang, "Some picture fuzzy Dombi heronian mean operators with their application to multi-attribute decision-making," Symmetry, vol. 10, no. 11, p. 593, 2018.

[41] F. Ates and D. Akay, "Some picture fuzzy Bonferroni mean operators with their application to multicriteria decision making," International Journal of Intelligent Systems, vol. 35, no. 4, pp. 625-649, 2020.

[42] S. Z. Luo and L. N. Xing, "Picture fuzzy interaction partitioned heronian aggregation operators for hotel selection," Mathematics, vol. 8, no. 1, p. 3, 2020.

[43] Y. Qin, Q. Qi, P. Shi, P. J. Scott, and X. Jiang, "Novel operational laws and power Muirhead mean operators of picture fuzzy values in the framework of Dempster-Shafer theory for multiple criteria decision making," Computers \& Industrial Engineering, vol. 149, p. 106853, 2020.

[44] S. Khan, S. Abdullah, and S. Ashraf, "Picture fuzzy aggregation information based on Einstein operations and their application in decision making," Mathematical Sciences, vol. 13, no. 3, pp. 213-229, 2019.

[45] G. Wei, "Picture fuzzy Hamacher aggregation operators and their application to multiple attribute decision making," Fundamenta Informaticae, vol. 157, no. 3, pp. 271-320, 2018.

[46] C. Jana and M. Pal, "Assessment of enterprise performance based on picture fuzzy Hamacher aggregation operators," Symmetry, vol. 11, no. 1, p. 75, 2019.

[47] L. Wang, H. Garg, and N. Li, "Pythagorean fuzzy interactive Hamacher power aggregation operators for assessment of express service quality with entropy weight," Soft Computing, vol. 25, no. 5, 2021.

[48] Z. Xu, "Choquet integrals of weighted intuitionistic fuzzy information," Information Sciences, vol. 180, no. 5, pp. 726-736, 2010.

[49] T. He, G. Wei, J. Wu, and C. Wei, "Qualiflex method for evaluating human factors in construction project management with pythagorean 2-tuple linguistic information," Journal of Intelligent \& Fuzzy Systems, vol. 40, no. 3, pp. 1-12, 2021.

[50] L. Fan, J. Lu, G. Wei, J. Wu, C. Wei, and Y. Guo, "GRA method for waste incineration plants location problem with probabilistic linguistic multiple attribute group decision making," Journal of Intelligent \& Fuzzy Systems, vol. 39, no. 3, pp. 2909-2920, 2020.

[51] A. Devaraj and P. Chellamani, "Picture fuzzy labelling graphs with an application," Annals of Optimization Theory and Practices, vol. 3, no. 3, pp. 117-132, 2020.

[52] M. Khan, S. Phiang Su Ngnoen, H. Rehman, and W. Kumam, "Applications of generalized picture fuzzy soft set in concept selection," Thai Journal of Mathematics, vol. 18, no. 1, pp. 296-314, 2020.

[53] M. Riaz, H. Garg, H. Muhammad Athar Farid, and R. Chinram, "Multi-criteria decision making based on bipolar picture fuzzy operators and new distance measures," Computer Modeling in Engineering \& Sciences, vol. 127, no. 2, pp. 771-800, 2021.

[54] H. Garg, "Exponential operational laws and new aggregation operators for intuitionistic multiplicative set in multipleattribute group decision making process," Information Sciences, vol. 538, pp. 245-272, 2020.

[55] U. Kifayat, M. Tahir, and J. Naeem, "Similarity measures for $\mathrm{t}$-spherical fuzzy sets with applications in pattern recognition," Symmetry, vol. 10, no. 6, p. 193, 2018. 